Portland State University

PDXScholar

7-1972

\title{
Simulation of Brain Damage on Bender-gestalt Test by College Subjects
}

Arnold Rahn Bruhn

Portland State University

Follow this and additional works at: https://pdxscholar.library.pdx.edu/open_access_etds

Part of the Psychology Commons

Let us know how access to this document benefits you.

\section{Recommended Citation}

Bruhn, Arnold Rahn, "Simulation of Brain Damage on Bender-gestalt Test by College Subjects" (1972). Dissertations and Theses. Paper 1580.

https://doi.org/10.15760/etd.1579

This Thesis is brought to you for free and open access. It has been accepted for inclusion in Dissertations and Theses by an authorized administrator of PDXScholar. Please contact us if we can make this document more accessible: pdxscholar@pdx.edu. 
AN ABSTRACT OF THE THESIS OF Arnold Rahn Bruhn, Jr., for the Master of Science in Psychology presented July 21, 1972.

Title: Simulation of Brain Damage on the Bender-Gestalt Test by College Subjects.

APPROVED BY MEMBERS OF THE THESIS COMMITTEE:

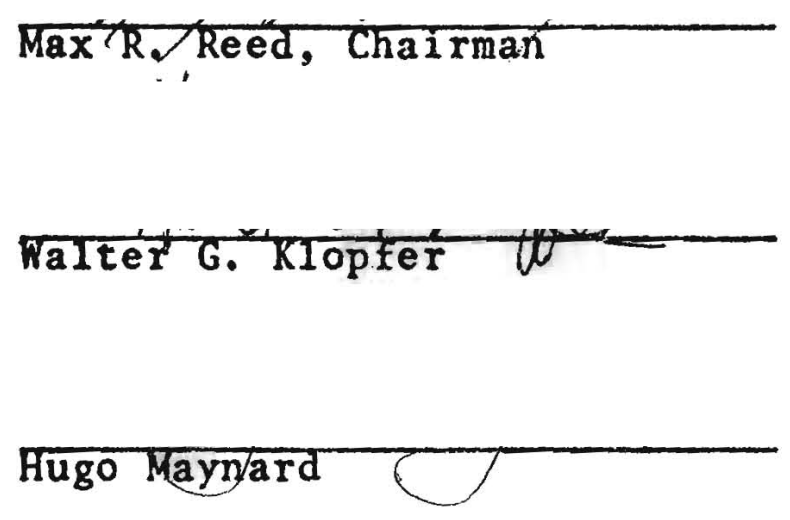

It is a frequent occurrence in accident cases involving alleged brain injury and attendant damage suits, for the defendant's attorney to charge the plaintiff in the case with malingering. At some time in the court proceedings it is also common that the plaintiff's psychologist will testify to the court that his client's brain damage is genuine, not feigned. but heretofore, clinicians have been able to speak to the court only from their own background of clinical experience, and clinicians have been known to disagree. They have not been able to refer to a body of research that has 
addressed itself to the question, Can Ss simulate brain damage on the Bender-Gestalt Test? This issue is particularly significant since many clinicians use the Bender to assess cases in which brain damage is suspected.

Since the thrust of this study was exploratory, the answer to this experimental question is not totally conclusive. However, it was determined that college Ss of at least normal intelligence who did not present evidence of brain damage on an initial Bender screening could not simulate traumatic brain damage. Although there is little reason to believe that non-organic Ss from other kinds of educational backgrounds (excluding, possibly, Ss who are extremely knowledgeable about the Bender), ages and levels of intelligence could simulate traumatic brain damage successfully, this possibility exists and should be researched.

The project consisted of a pilot study and a main study. In the pilot study 18 volunteers from an upper division psychology class were tested with a Bender in a group situation under standard instructions. Four weeks later they were asked to simulate brain damage on the Bender. The test records of 18 organic Ss were used to form a criterion group. An ABPP certified clinical psychologist then attempted to sort the malingerers from the organics (Sort 1) and the normals from the organics (Sort 2). On the first sort, 2 of the $18 \mathrm{Ss}$ in each group were missorted. On the second sort, 1 organic and 2 normals were missorted. The resulting Phi coefficients were .78 and .83 , respectively. Since existing 
quantitative sorting methods (i.e., the Pascal-Suttell system and the (anter system) were not able to validly differentiate malingerers from organics, it became necessary to spell out the implicit criteria which the clinician used as he performed his sorts. An analysis of the criteria then revealed that all of the missorts, except 1 organic $S$, involved confusions of "normal" Ss who were either borderline organics or mildly organic with organic Ss who were mildly organic. The main study was necessary to replicate the findings obtained from the pilot study since the criteria devised therefrom were formulated 'ex post facto.' The results obtained were generally comparable to those from the pilot study. In the main study, a second clinician independently sorted the groups in order to provide a reliability check on the sorting procedure. An analysis of the sorting decisions resulting from the criteria-based sorting procedure indicated that the sorting decisions of the 2 clinicians concurred on 84 of the protocols in the malingerer-organic sort and on 948 of the records in the normal-organic sort. A sort-resort operation by the first clinician on the malingerer-organic sort with one day intervening resulted in 100 agreement between his judgments. 
SIMULATION OF BRAIN DAMAGE ON THE BENDER-GESTALT

TEST BY COLLEGE SUBJECTS

\author{
by \\ ARNOLD RAHN BRUHN, JR.
}

A thesis submitted in partial fulfillment of the requirements for the degree of

MASTER OF SCIENCE in PSYCHOLOGY

Portland State University 1972 
TO THE OFFICE OF GRADUATE STUDIES:

The members of the Committee approve the thesis of Arnold Rahn Bruhn, Jr., presented Ju1y 21, 1972.
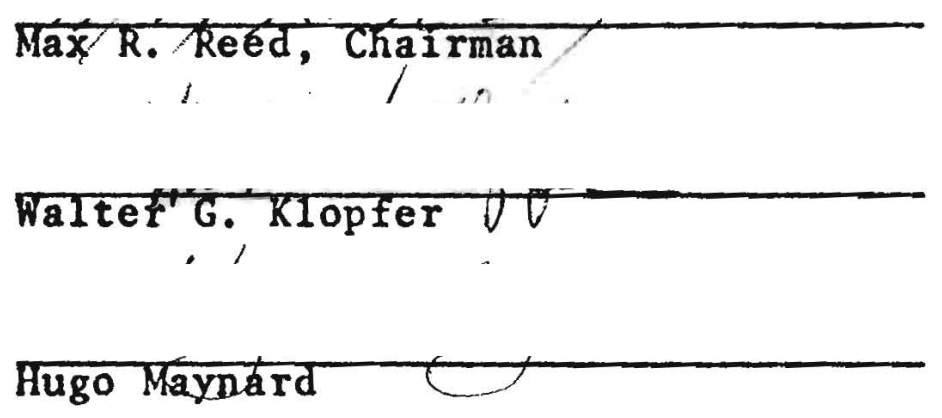

\section{APPROVED:}

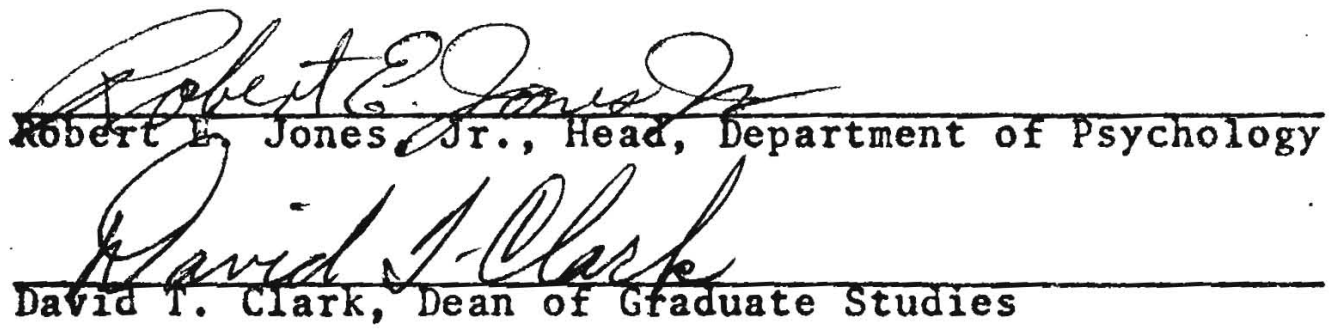

July 21, 1972 


\section{ACKNOWLEDGEMENTS}

When Max Reed introduced me to the Bender-Gestalt Test nine months ago, I could little appreciate the toil which loomed ahead. However, as my involvement in the research grew, I came to appreciate through his timely comments some of the beauty and potential of this test. His dedication to this project and the many hours of time he freely gave deserve more eloquent words of thanks than I could hope to express. But most of a11, Max, thanks for your patience, encouragement and kindness.

Gratitude is also due the many members of the psychology department who reviewed the design of the experiment and contributed their suggestions; to Walter Klopfer and Hugo Maynard, members of my committee; and especially to Jim Paulson, statistics expert par excellence, who generously shared his mathematical wisdom. Special thanks go to my wife, Arlene, and to Chris Mazuca for their assistance in preparing this manuscript. 
TABLE OF CONTENTS

PAGE

ACKNOWLEDGEMENTS • • • • • • • • • • • • •

LIST OF TABLES . . . . . . . . . . . . vii

CHAPTER

I INTRODUCTION . . . . . . . . . . 1

Research Hypothesis ... . . . 1

II A REVIEW OF THE LITERATURE AND A

DESCRIPTION OF THE RESEARCH

PROJECT . . . . . . . . .

History of Simulation Studies with

the Bender-Gestalt .......

The Measurement Function of the Bender-Gesta1t .......

Quantitative Scoring Systems and the Bender Test ........

Group Vs. Individual Testing on the Bender.......... 8

Selection of Subjects ...... 9

Balancing Subject Groups ..... 10

Research Design . . . . . . . 11

Pilot Study Main Study 
II RESULTS •. . . . . . . . . . . 16

Pilot Study . . . . . . . . . 16

Main Study .. . . . . . . . . 18

IV DISCUSSION . . . . . . . . . . . . 22

Pilot Study . . . . . . . . . 22

The Presence of Organic Ss in a

Sample of College Students

Drug Use and Figural

Distortions

Strong and Weak Organic

Signs

Scoring Errors: Possible

Explanations

Errors Within the Group of College Ss Classified as "Borderline" or "Mild1y Organic"

Errors Within the Organic Criterion Group

The Ability of Quantitative Scoring Methods to Make Valid Sorting Decisions

The Ability of the Pilot Study to Make Valid

Differentiations

Main Study . . . . . . . . . . 36

The Questionnaires

Further Studies

Limitations of the Study

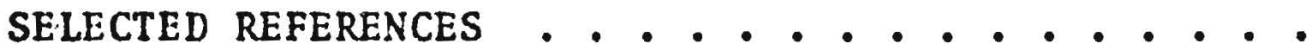



A. QUESTIONNAIRES
B. DIFFERENTIAL DIAGNOSTIC CRITERIA
c. CHECKLIST FOR MALINGERING
D. CROSS CHECKS FOR DISTORTION CONSISTENCY
E. SAMPLE ORGANIC AND MALINGERER PROTOCOLS
F. THE BENDER-GESTALT TEST FIGURES 


\section{LIST OF TABLES}

TABLE

PAGE

I Sorting Results . . . . . . . . . 17

II Distribution of Pascal-Suttell Scores

in the Pilot Study . . . . . . . . 19

II Distribution of Canter Scores in the

Pilot Study ........... . 20

IV Canter Scores for College Students Under

Standard Instructions and

Malingering Instructions ....... 


\section{CHAPTER I}

\section{INT RODUCT ION}

In court cases which involve damage suits and injury claims alleging brain damage, the defendant's attorney will frequently accuse the plaintiff of malingering or at least intimate that his veracity is suspect. In such cases clinical psychologists are often called by the lawyers of both parties as expert witnesses who will address themselves to the issue of simulation; unfortunately, sometimes the experts disagree. Although little research has been done on simulation with regard to brain damage, the results of several investigations suggested that it is difficult for "normal" Ss to simulate insanity or retardation. Thus, as the project began, it seemed reasonable to assume that non-braindamaged Ss would be unable to simulate brain damage.

\section{RESEARCH HYPOTHESIS}

The project was designed to test the hypothesis: Naive Ss of at least normal intelligence who have not sustained brain trauma cannot simulate brain damage on the Bender-Gestalt Test. 
CHAPTER II

\section{A REVIEW OF THE LITERATURE AND A DESCRIPTION OF THE RESEARCH PROJECT}

The general approach of this chapter is to discuss the existing research findings, whenever possible, in terms of their implications for this project. However, those findings which could be classified as historical background, as distinct from those findings which seem to bear directly on the project itself, are discussed in the first section. Next, general Bender topics are discussed in the three subsequent sections, usually in terms of their relevance to this project. In the remaining sections, specific issues which directly pertain to this project are detailed, the design of the project is discussed, and specific findings in the previous research are cited when appropriate.

\section{HISTORY OF SIMULATION STUDIES WITH THE BENDER-GESTALT}

Before discussing the previous simulation studies, one should note first that little work has been done in the area, and that the little work which has been done is exploratory in nature. Bender (1938) instructed physicians, medical students and nurses who were not familiar with the principles 
of the Gestalt function to take the Bender-Gestalt Test and to as sume

... either that they were mental defectives or that they were in a situation whe re they tried to pretend that they were mental defectives and not able to draw the figures in the correct way. (p.150)

She also tested prisoners who had been or were being tried for criminal offenses and who seemed to be simulating a psychosis or who presented a Ganser syndrome. However, the absence of a control group weakened her findings. Further, in the first example cited above, Bender was aware that a11 her Ss were simulators. Since standard research methods were not used, Bender's conclusion should be regarded at best as suggestive:

... none of these individuals (i.e., doctors, medical students, nurses, or prisoners) was successful in neglecting the essential Gestalt principles which their maturational level would make it possible for them to experience. (p. 150)

Nevertheless, her findings suggest that it would be difficult for an individual to simulate mental deficiency or psychosis. Blum \& Nims (1953) obtained similar results when they compared the Bender-Gestalt Test records of 35 neuropsychiatric hospitalized servicemen with those of 35 randomly selected enlisted men who were instructed to feign mental illness. Although the results indicated that the two groups could not be differentiated using the Pascal-Suttell scoring system, two clinical psychologists succeeded in sorting the 
protocols at a statistically significant level $(p=.01)$.

The records of the "feigners" indicated the following patterns: one geometric pattern was sometimes substituted for another; more complex drawing elements were added; and figures were converted into simple symbols. However, since 25 of the 70 Ss were misgrouped, one should regard the findings with caution.

\section{THE MEASUREMENT FUNCTION OF THE BENDER-GESTALT}

Theoretical assumptions concerning what Ss do on the test were given by Bender in her 1938 monograph:

The Gestalt function may be defined as that function of the integrated organism whereby it responds to a given constellation of stimuli as a whole; the response itself being a constellation, a pattern, or a Gestalt. (p. 3)

She further stated:

The whole setting of the stimulus and the whole integrative state of the organism determine the pattern of the response. (p. 4)

Pascal \& Suttell (1951) observed:

If we accept as given the ability to perceive and execute the designs, deviant performance should, then, be a function of the interpretative factors which obtrude between perception and execution. ( $p$, 6) 
Specifically, with regard to brain damage, the same writers stated:

With increasing damage to the cortex there seems to be greater disturbance in reproductive capacity resulting in primitivation of the forms and destruction of the Gestalten.... Reproductions resulting in fragmentation, destruction or primitivation of the Gestalten are regularly obtained in children below the age of six, from patients immediately after convulsive the rapy, from brain-damaged individuals, paretics, and seniles; in other words wherever there is cortical deficit or damage. (p. 8)

Since normal naive $S s$ would have little idea of how to fragment, destroy, or render more primitive the Gestalten, or even know they should do so, it seems unlikely that they could, in fact, simulate brain damage. In this regard

Pascal \& Suttell (1951) specifically stated:

Adults of normal intelligence without known cortical damage do not, in our experience, fail to reproduce the essential Gestalten. (p. 8)

III. QUANTITATIVE SCORING SYSTEMS AND

THE BENDER TEST

Empirical research with the Bender-Gestalt was hindered in its early history by a lack of objective scoring methods. The publication of a reliable, "standard" scoring method by Pascal \& Suttell in 1951 greatly facilitated research with the test. Although adequate norms for some age groups were not established by the authors, ample normative information was available for the $S$ s used in the research project. 
A review of the literature, however, indicated that there were problems associated with the use of the PascalSuttell scoring system in identifying simulators. Specifically, Blum \& Nims (1953) achieved poor results with the system while attempting to distinguish psychiatric patients from presumably normal, randomly selected enlisted men who were instructed to feign mental illness. Thus, it seemed logical that the Pascal-Suttell scoring system would probably be of little value in discriminating braindamaged Ss from simulators.

However, the parallel between the present study and the Blum \& Nims study was not exact. Blum \& Nims did not establish base line Pascal-Suttell scores on the BenderGestalt with their simulator group before the Ss were instructed to simulate; thus, it was possible, as the authors pointed out, that the simulators were not "normal," as had been assumed. Further, they pointed out that the mentally ill patients in the sample could have been simulating. Therefore, it was possible, although not likely, that the selection of the Ss resulted in confounded groups and thus, the ineffectiveness of the Pascal-Suttell scoring system would be explained. Further, Ss in the present study were to simulate brain injury, not mental illness; thus, it was possible that the scoring system might effectively separate simulators of brain damage from organics even if it was true that the system did not separate simulators of mental illness from mentally ill Ss. One of the purposes of the 
pilot study in the present project was to assess the value of the Pascal-Suttell scoring system in distinguishing simulators from organics.

It seems appropriate to observe at this point in the discussion that the principal reason the Pascal-Suttell scoring system has been so ineffective in identifying simulators is that the scoring system is a cumulative, quantitative measure of undifferentiated abnormal distortions of the stimulus designs. Thus, any distortions of the essential Gestalten in the designs is scored. Predictably, then, simulators often score as high or even higher than comparison groups of psychiatric patients or retarded patients when the Pascal-Suttell scoring system is used. However, specific disorders are characterized by particular and consistent patterns of distortions on the Bender-Gestalt, not simply by any distortion. This argument especially applies to patients with organic brain disorders. Thus, a scoring system which seeks to distinguish malingerers from organics must weight heavily those distortions which are consistently produced by an organic criterion group and are rarely produced by malingerers. Further, those distortions atypical of organics but common to simulators would be assigned a negative weight.

Since the Pascal-Suttell system was not designed specifically to distinguish normals or simulators from organics, it was likely that the system would prove useful at best only as a screening device during base line testing. 
Such a screening was necessary in order to determine whether organic Ss were present in the simulator group. However, it was not clear whether the Pascal-Suttell system would be more effective than other scoring systems, such as Arthur Canter's scoring method (1966), which was designed specifically to identify Ss with brain damage. Another purpose of the pilot study, then, was to determine which of the two systems worked more efficiently.

But neither the Pascal-Suttell nor the Canter system was intended to be used to separate malingerers from organics. Thus, a further purpose of the pilot study was to determine whether either of these scoring systems would be suitable for that use. Assuming the failure of both systems to differentiate the two groups, and assuming the success of experienced clinicians to make such differentiations in a sorting operation, the writer hoped that he could devise either a quantitative or a descriptive system which could be used to separate the two groups. In the event that an alternative system proved necessary, the system could be validated in the main study.

IV. GROUP VS. INDIVIDUAL TESTING ON THE BENDER

Another issue to be considered is the effect of group vs. individual testing on Bender-Gestalt performance. Blum \& Nims (1953) found that the Pascal-Suttell scores of Ss who were tested both individually and in a group situation did not differ significantly. Given Blum \& Nims' finding, 
it was felt that group testing was justifiable in this study. The size of the cards used in this study, including design and margins, was $10-5 / 8$ inches by $15-1 / 2$ inches, the same as that used by Blum \& Nims.

\section{SELECTION OF SUBJECTS}

Since this research project sought to discover whether non-braindamaged Ss could simulate brain damage on the BenderGestalt, it was imperative that the Ss in the experimental group not have sustained brain damage. But since EEGs were too expensive to administer to each $S$, and since EEGs do not provide a definitive test for brain damage anyway, it was reasoned that the problem would be dealt with if a sample of college students in psychology classes were used. In all likelihood, grossly braindamaged Ss would not be able to function in the college setting and, therefore, would not appear in the sample. But in order to eliminate Ss who might have been able to function academically in spite of an organic handicap, a series of questionnaires (see Appendix A) was used subsequent to all testing to attempt to determine whether the Ss had sustained cortical trauma. Although not a definitive resolution to the problem, the questionnaires provided a means of eliminating $S s$ who were aware of such damage and/or cortical difficulties (and were willing to admit to same) but who were not screened out by academic demands or by an initial screening with the Bender-Gestalt. Since college students as a group would be superior in intelligence to a 
normal population, it seemed reasonable that college students would be better simulators than a random sample of a normal population, assuming that ability to simulate and intelligence are positively correlated. Thus, a sample of naive college students should have provided a more rigorous test of the hypothesis used in the study. However, since little is known about simulation within the context of the present study, this argument is speculative. Test records of organic Ss used in the pilot study were obtained through a local clinician; records of organic Ss used in the main study were obtained from the Portland Veterans Administration Hospital, from the Rehabilitation Institute of Oregon and from several clinicians.

\section{BALANCING SUBJECT GROUPS}

Pascal \& Suttell (1951) found that Bender-Gestalt scores are not affected when Ss have normal or better intelligence and fall within an age range of 15-50 years; that test scores are not related to age, at least between ages 15-50; and that test scores are not significantly related to the sex of a $S$. Therefore, with two exceptions, the $S$ groups were not equated for these variables. First, since normative studies were incomplete (due to small n's) outside an age range of 15-50 years, the $S s$ in the experimental groups and the organic control groups were excluded if they fell outside this age range. Second, Ss in al1 groups had to have at least average intelligence (i.e., 90 or 
better). Again, it should be stressed that the study only required an experimental group of "non-organics;" therefore, balancing $S$ groups for intelligence, age and sex was not deemed necessary. Pascal \& Sutte11 (1951) also found a correlation of .38 between education and Bender-Gestalt scores. Specifically, Ss with at least some college education tended to have lower deviation scores than Ss with one or more years of high school when ss were equated for age. This finding was interpreted to suggest that college-educated Ss might attend more carefully to perceptual details and therefore might simulate more effectively than non-college educated Ss. The finding also suggests that one might expect to find fewer braindamaged $S s$ in a college-educated group, an implication which makes intuitive sense. However, both inferences are merely speculative.

\section{RESEARCH DESIGN}

The project consisted of a pilot study and a main study, described below.

\section{Pilot Study}

The experimental group of malingerers consisted of 18 volunteer Ss from an upper division psychology class. The Ss were given a group Bender-Gestalt with cards previously described which contained enlarged versions of the standard test designs. Their directions were as follows: "I am going to show you some cards which have some simple designs 
drawn on them. There will be nine of them, and I will show them to you one at a time. I want you to copy them freehand without sketching. You will have two minutes to copy each drawing. Do not turn your paper. You will do all your designs on one sheet of paper." Including instructions, the testing period lasted 25 minutes. Four weeks later, the Ss took the test again, but this time they were to feign brain damage. The instructions were as follows: "I am going to show you the same cards again. You will recall that there were nine of them. You will be shown one card at a time for two minutes. You will copy them without sketching. Do not turn your paper. This time, however, I want you to copy the designs the way you believe that a person with brain damage would copy them." The final instruction elicited groans, laughter and several pleas for specificity; e.g., "What kind of brain damage?" Questions of this genre were responded to non-committally. For example, "That's up to you." Ss seemed to be quite involved in the test, despite their initial reaction of incredulousness, and many expressed disappointment when they were told that the test results could not be released until summer, 1972. Following the second test, a series of questionnaires (reproduced as Appendix A) was administered in sequence. The purposes of these questionnaires were as follows: 1) to determine whether the Ss had deduced the purpose of the experiment; 2) to determine whether any of them were aware of any brain injury which they might have sustained; 3) to determine 
what they knew about the Bender-Gestalt and specifically, what kinds of distortions they thought that a braindamaged person might produce on the test. The total time required during the second testing was approximately 33 minutes. Next, a control group consisting of 18 records from organics with medically verified brain damage was randoinly selected from the files of a local clinical psychologist. Then, the test records of the college students under malingering instructions and the records of the organic patients we re shuffled, and a sorting procedure (Sort 1) was performed by an ABPP certified clinical psychologist. This procedure was repeated with the records of the college students under standard instructions and the protocols of the organic Ss (Sort 2). The tests were then scored by the Pascal-Suttell method and by the Canter scoring system (Canter, 1966, pp. 5-28). Concerning the latter system, only the scoring weights were used; deviation scores and Background Interference Procedure (BIP) paper were not used. Since neither system was capable of satisfactorily differentiating malingerers from organics, the protocols of organics and malingerers were studied in order to discover what kinds of distortions malingerers produced which organic Ss never, or very rarely, made. The purpose of this procedure was to devise an objective means of identifying malingerers in the main study through a set of differential criteria derived from the pilot study (see Appendices B, C and $D)$. 
Main Study

This time the experimental group consisted of 20 volunteer Ss from an upper division psychology class. The previously described test conditions, instructions and procedures were followed as before with two exceptions. First, the retest with malingering instructions followed the initial test with standard instructions by four days, in contrast to the four-week interval used in the pilot study. Second, the last sentence in the malingering instructions (see p. 12) was deleted, and the following statement was inserted: "This time, however, I want you to pretend that you have sustained brain damage in an accident (for example, auto or industrial) and that you are suing for large damages as a compensation for your injuries. Although you did, in fact, sustain a concussion and although you have had periodic headaches, your doctors have not been able to detect through current medical tests any evidence of specific brain tissue damage or any evidence which indicates that you will suffer any permanent impairment or damage to your faculties. Nevertheless, you press your case, determined to collect damages. The test you are about to take today will be crucial. You have reason to believe that the results of the test will bear heavily on the outcome of your case. Therefore, you are resolved to do anything, even fake the test, to win your case." The reason for the increased specificity in instructions was to direct the Ss to simulate, as best 
they could, a brain injury which might have resulted from an external blow to the skull. This type of injury often results in certain patterns of distortions on the Bender which are distinct from other kinds of organic distortions (e.g., such as those present on the records of epileptics and stroke victims).

With regard to the organic Ss, the criterion of brain damage used in this study was as follows: there must have been a traumatic type injury to the brain resulting from an external blow to the skull, and the trauma must have been verified medically. Thus, cerebral palsy patients and chronic alcoholics, to cite two examples, were eliminated from the organic sample. The reason for selecting this criterion was that most cases of alleged malingering which the clinician encounters result from accident cases. As described previously in the pilot study, the protocols were shuffled by the author and independently sorted according to the criteria developed in the pilot study by two clinicians. Estimates of inter-rater agreement and sort-resort stability of judgments by the same clinician were obtained on the malingerer-organic sort. 
CHAPTER III

\section{RESULTS}

Although the results of the pilot and main studies generally substantiated the research hypothesis, two simulators who appeared to be organics judging from their Bender performance under standard instructions were able to feign brain damage successfully in the pilot study on the Bender-Gestalt Test.

\section{PILOT STUDY}

In the pilot study the ABPP certified psychologist correctly sorted 16 malingerers and 15 organics from the $18 \mathrm{Ss}$ in each group. Stated in percentages, 898 of the sorts were correct. The Phi coefficient (from Hays, 1963, p. 604) thus obtained was .78 (see Table I). The same sorting procedure was applied to the normal $(n=18)$ and organic $(n=18)$ groups. This time 1 organic $S$ was misclassified as normal, and 2 normals were grouped with the organics. A total of 92 of the sorts in the second sorting procedure, or 33 of 36 , were correct. The resulting Phi coefficient was .83 . Relative to the sorting results, the quantitative methods employed fared poorly by comparison. 
TABLE I

SORTING RESULTS

Clinician 1 *

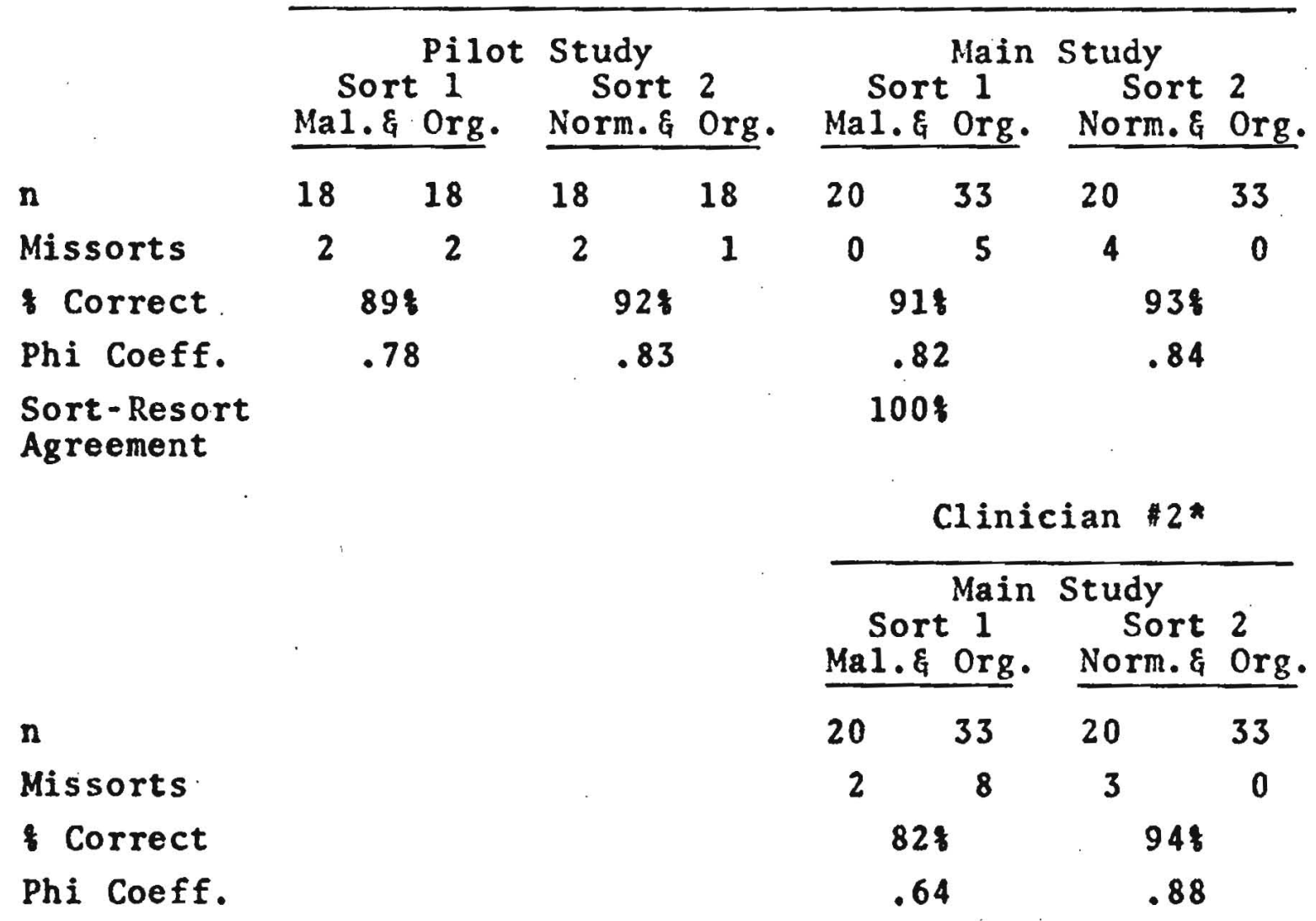

* Clinician 1 helped to devise the pilot study criteria. His background included considerable recent and past experience in testing organic Ss on the Bender. Clinician 2 had had no recent experience with testing organic Ss on the Bender, so the pilot study records were used for training purposes to illustrate the sorting criteria. Subsequent to sort 1 , clinician 2 observed that many of his mistakes resulted from an inadequate mastery of the criteria-specifically, how to sort a protocol which contained both malingerer signs and organic signs. 
Using a cutoff score of 9 , the Pascal-Sutte1i scores correctly identified 11 of 18 normals without misgrouping any organics. But the scoring method could not separate malingerers from organics (see Table II). In comparison, the Canter scoring method seemed to be slightly more powerful. Using a cutoff score of 9,14 of 18 normals were correctly identified, and no organics were incorrectly labeled as normal. Using a cutoff score of 13 , one would correctly identify 17 of 18 normals, but 2 organics would be falsely labeled as normals. A cutoff score of 9 also identified 3 of the 18 malingerers without including any organics. An analysis of the remaining scores indicated that no gain would accrue from raising the cutoff score. Essentially, then, the Canter system seemed to sort out organics from normals rather we11, but it failed to provide the hoped for quantitative differential diagnostic method which would distinguish malingerers from organics (see Table III). Thus, it became necessary to spell out the implicit criteria used by the clinician as he sorted the organics from the malingerers (see Appendices B, C and D). Although these criteria worked quite well in the pilot study, the main study became necessary to replicate the procedure.

\section{MAIN STUDY}

In the main study, the clinicians used the pilot study criteria (see Appendices B, C and D) as they performed the sorting operation. In the malingerer-organic sort, 0 of the 


\section{TABLE II *}

DISTRIBUTION OF PASCAL-SUTTELL SCORES

IN THE PILOT STUDY

\begin{tabular}{|c|c|c|c|}
\hline $\begin{array}{c}\text { Test } \\
\text { Score } \\
\text { Interval }\end{array}$ & $\begin{array}{l}\text { Normal } \\
\text { Group }\end{array}$ & $\begin{array}{l}\text { Ma1 ingerer } \\
\text { Group }\end{array}$ & $\begin{array}{c}\text { Organic } \\
\text { Group }\end{array}$ \\
\hline $0-9$ & 11 & 0 & 0 \\
\hline $10-14$ & 1 & 1 & 1 \\
\hline $15-19$ & 0 & 3 & 1 \\
\hline $20-24$ & 1 & 1 & 0 \\
\hline $25-29$ & 1 & 1 & 1 \\
\hline $30-34$ & 2 & 1 & 4 \\
\hline $35-39$ & 1 & 1 & 1 \\
\hline $40-44$ & 1 & 5 & 0 \\
\hline $45-49$ & 0 & 0 & 3 \\
\hline $50-54$ & 0 & 1 & 2 \\
\hline $55-59$ & 0 & 0 & 1 \\
\hline $60-69$ & 0 & 2 & 1 \\
\hline $70-79$ & 0 & 0 & 1 \\
\hline $80-89$ & 0 & 1 & 1 \\
\hline \multirow[t]{2}{*}{90.99} & 0 & 1 & 1 \\
\hline & $n=18$ & $\mathrm{n}=18$ & $n=18$ \\
\hline
\end{tabular}


TABLE III

DISTRIBUTION OF CANTER SCORES IN THE PILOT STUDY

\begin{tabular}{|c|c|c|c|}
\hline $\begin{array}{c}\text { Test } \\
\text { Score } \\
\text { Interval }\end{array}$ & $\begin{array}{l}\text { Norma 1 } \\
\text { Group }\end{array}$ & $\begin{array}{l}\text { Mal ingerer } \\
\text { Group }\end{array}$ & $\begin{array}{c}\text { Organic } \\
\text { Group }\end{array}$ \\
\hline $0-4$ & 8 & 1 & 0 \\
\hline $5-9$ & 6 & 2 & 0 \\
\hline $10-13$ & 4 & 2 & 2 \\
\hline $14-19$ & 0 & 1 & 3 \\
\hline $20-24$ & 0 & 3 & 5 \\
\hline $25-29$ & 0 & 0 & 1 \\
\hline $30-34$ & 0 & 3 & 0 \\
\hline $35-39$ & 0 & 1 & 2 \\
\hline $40 \cdot 44$ & 0 & 0 & 0 \\
\hline $45-49$ & 0 & 1 & 1 \\
\hline $50-54$ & 0 & 1 & 1 \\
\hline $55-59$ & 0 & 1 & 0 \\
\hline $60-64$ & 0 & 0 & 1 \\
\hline $65-74$ & 0 & 1 & 1 \\
\hline $75-125$ & 0 & 0 & 0 \\
\hline 126 & 0 & 1 & 0 \\
\hline 176 & $\underline{0}$ & $\underline{0}$ & 1 \\
\hline & $n=18$ & $n=18$ & $n=18$ \\
\hline
\end{tabular}


20 malingerers and 5 of the 33 organics were missorted by the first clinician, leaving 48 of the 53 sorts correct, or 918. The resulting Phi coefficient was .82 . In the normalorganic sort, 4 of the 20 normals and 0 of the 33 organics were misclassified by the first clinician, leaving 49 of the 53 sorts correct, or 938 . The Phi coefficient thus obtained was .84 . The judgments of the 2 clinicians agreed on 848 of the protocols in sort 1 (their classifications differed on 2 malingerer and 7 organic records) and on 948 of the cases in sort 2 (their classifications differed on 3 normal records). Sort-resort agreement between the judgments of clinician 1 on the first sort was 100 when an interval of one day separated the two sorts. 
CHAPTER IV

DISCUSSION

I. PILOT STUDY

Several interesting findings came from the pilot study. These findings can be discussed within the context of the following topics: 1) the assumption that organic Ss would be "screened out" naturally by academic demands in a sample of college Ss; 2) possible explanations for the clinician sorting errors; 3) the ability of the Pascal-Suttell system and the Canter system to make valid sorting differentiations; and 4) the ability of the pilot study criteria to make valid differentiations in the sorting operations.

The Presence of Organic Ss in a Sample of College Students

It was initially assumed that few organic Ss would be found in a sample of college students. The test results on the Bender-Gestalt under standard instructions showed that only 11 Ss had $0-2$ organic signs, as previously defined by the criteria in the appendices. A total of 4 Ss were "borderline" organics, and 3 records 
indicated "mild" organic damage." The questionnaires used supported, at least in several cases, our conclusion that some of these Ss had sustained brain damage. ${ }^{2}$ But before the criteria in the appendices were formulated (an operation which was carried out subsequent to the two sorting operations), a decision had to be made as to whether some, or all, of the Ss with definite signs of organic involvement should be excluded from the sample. The argument for exclusion was powerful: it made no sense to ask Ss who seemed to be organic to feign organic damage. Obviously, such an S. had the option of simply doing what he had already done, which would not

${ }^{1}$ For purposes of discussion, a $S$ was judged to have "borderline" organic damage if the distortions in the gestalt were minor (or "weak"), relative to the control group of organics, yet still indicative of organic involvement. A S was judged to have "mild" organic damage if the distortions in the gestalt were generally "stronger" or more pronounced (and usually greater in number), relative to those appearing in "borderline" records. Ss in the "mild" group usually came very close to entirely losing the gestalt on at least one design, most often Design 3 , and at least 2 other designs were poorly drawn in a manner typical of organic Ss. In such records, erasures, second at tempts and/or workovers were frequent, indicating that the $S$ experienced considerable difficulty in executing even a poorly drawn design. One may correctly assume that there was considerable overlap between the overall design quality in the records of these $3 \mathrm{Ss}$ and the design quality apparent in the records of organics with the same classification. This overlap in design quality between the two groups was probably responsible for the sorting errors in the normal-organic sort, and, to a lesser extent (in this sample), for the sorting errors in the malingerer organic sort.

${ }^{2}$ That is, several ss supplied information in the questionnaires which indicated that brain damage had been, or might have been sustained in the past. For example, various Ss mentioned auto accidents which caused head injuries, high sustained fevers, and falls which resulted in severe head injuries. 
constitute a fair test of the hypothesis. In the end a compromise was effected. It was decided to retain in the pilot study all 7 of the Ss in the two debatable groups for several reasons: 1) all but the $3 \mathrm{Ss}$ in the "mild" group could be sorted out on the basis of less severe distortions relative to the organic controls; 2) the 3 Ss in the "mild" group did not provide any grounds for exclusion according to information supplied in their questionnaires; 3 ) it could not be decided whether distortion evidence, questionnaire evidence, or both kinds of evidence were necessary for exclusion before the pilot study was completed; 4) we were curious to discover what would happen if we included all Ss in the pilot study. As a result of the pilot study, we concluded that "mild" organic Ss in the "normal" sample should be excluded from the main study, regardless of questionnaire information. ${ }^{3}$. The latter was to be regarded only as information which supported the exclusion decision if it indicated that organic involvement was likely. Obviously, it would have been desirable to attempt to

3 The rationale for keeping "borderline" cases in the "normal" sample was that such Ss could be distinguished from the mild organics in the organic sample according to the pilot study criteria. This rationale proved faulty in the main study, however, when a number of even milder organic cases appeared in the organic sample. The retention of borderline records in the main study probably explains why 4 "normal" Ss ("borderline" organics by the pilot study criteria) were grouped with the organic Ss. In subsequent studies, "borderline" organics should be excluded from a sample of normals because the normal and organic groups might become confounded. 
substantiate the assumption of organic damage in such cases in which a "normal" $S$ was excluded, but the requisite resources were not available for such a followup. Drug Use and Figural Distortions. Three months after the pilot study was completed, a conversation with one $S$ in the experiment indicated that one explanation for the large number of organic signs in the records of pilot study ss might be drug involvement. She stated that she knew of at least $4 \mathrm{Ss}$ in the experiment who were then, at the time of testing, daily users of halucinogenic drugs. It then seemed significant in retrospect that several Ss asked me whether I was a "narc" when they filled out the questionnaires. Several Ss also stated verbally that they would not incriminate themselves by describing in writing their involvement with drugs. However, the argument that the organic signs observed in some of the more distorted records were at least partly a function of drug involvement is only speculative.

Strong and Weak Organic Signs. The term, "organic sign," as used previously in conjunction with the concepts "strong" and "weak" (see footnote 1) deserves some comment. Those familiar with the Pascal-Suttell and Canter scoring systems know that a given distortion on a given design is usually scored or not scored. Thus, we are essentially confronted with a "yes or no" decision, where a "yes" judgment on the question, "Is distortion X present?", is 
paired with a fixed predetermined weighted score. Generally, these scoring systems do not attempt to deal with the degree of a distortion. If one accepts the premise that distortions do differ in degree, and that a competent Bender-Gestalt tester can distribute distortions which differ widely in degree along a continuum, then it is apparent, for example, that 3 organic signs on $A^{\prime} s$ test record may not be equivalent in degree to 3 organic signs on B's record. Thus, the phrase, "strong organic signs," implies that these distortions are equivalent to those commonly found on records of organics with at least mild medically verified damage. On the other hand, it should be stressed that most of the organic signs found in the records of "normals" and "borderline organic" Ss in the normal $\mathrm{S}$ sample were not equivalent in degree (and usually in number also) to those commonly found in the records of organics with at least confirmed "mild" involvement. Accordingly, the term "weak" was applied to those organic signs which were not equivalent in degree to comparable signs found in records of organic Ss who presented evidence of at least minimal "mild" organic involvement.

Scoring Errors: Possible Explanations Errors Within the Group of College Ss Classified as "Borderline" or "Mildly Organic." A second finding from the pilot study was less unexpected. Of the 3 "normal" Ss who were previously labeled as "mild organics" according to the criteria used, 2 evidenced sufficiently strong organic 
signs to be misclassified as organic on the normal-organic sort. And 1 of these 2 was also misclassified on the malingerer-organic sort. The second "normal" misclassified on the malingerer-organic sort was labeled a "borderline organic" according to the criteria previously described. One might infer from the pilot study results, then, that Ss with the strongest organic signs on the standard instruction Bender-Gestalt Test would have the highest probability of being misclassified as organics on the malingerer-organic sort. Given the small number of such cases, it is impossible to make a definitive statement, but comments from the clinician-sorter indicated that records from these 7 dubious normals causes him the greatest amount of difficulty as he carried out the two sorting procedures. But one might well ask why the 3 "normal" Ss who were classified as "mild" organic cases didn't have even better success as malingerers. A review of their records indicated that one was guilty of "over-acting." But the other 2 Ss (as well as 2 of the "borderline" Ss) acted as if they did not know what to do, so they did more or less what they had done before as they rendered their drawings. Thus, since organic signs were still very much in evidence in their malingering records, these Ss presented considerable diagnostic problems for the clinician.

Errors Within the Organic Criterion Group. An analysis of the 3 organic records which were misclassified showed that 2 were of the "mild" variety. The third organic 
record showed many strong organic signs, but the figures were drawn with an apparent ease and regularity that is rare with organic Ss and relatively common with malingerers. As one might expect, this record was correctly classified in the normal-organic sort but was misclassified in the malingererorganic sort. Again, one can draw no firm conclusions from an analysis of this data due to the small number of cases and the 'ex post facto' method used, but the implications are clear.

The Ability of Quantitative Scoring Methods to Make Valid Sorting Decisions

A third finding, that the Pascal-Suttell and the Canter scoring systems did not successfully differentiate malingerers from organics, was also not unexpected. Neither system was designed to identify malingerers. Further, Blum \& Nims' findings (1953) suggested that the Pascal-Suttell scoring system was vulnerable to malingering of all types, since it scored distortions associated with numerous types of disorders. But, for the moment, let us assume that the $7 \mathrm{Ss}$ in the "borderline" and "mild" groups had been excluded from the study initially for the reasons previously mentioned. Using the same cutoff score as before, one finds that the number of missorted normals which were classified as organic would be reduced under each scoring system (only 2 of 11 by the Pascal-Suttell method and 0 of 11 by the Canter system), but neither system would have been helped in the crucial 
malingerer-organic sort. Since the Canter system was designed to deal more specifically with organic damage, it seems puzzling at first glance why the Canter system failed to distinguish malingerers from organics. At least two explanations are possible. First, Canter's BIP, which was not used in this study, might have distinguished malingerers from organics, even though his scoring system did not. However, this explanation is unlikely since the BIP scores are derived from Canter's scoring system. Second, the Canter system scores many distortions which are commonly produced by malingerers and organics alike. Evidence for the second explanation is provided by the summary of statistical data in Table IV.

A cursory glance at the test scores of Subject 2 is perhaps sufficient to make the point. Under malingering instructions, he scored 115 points higher than under standard instructions, conclusive evidence that the Canter system is not immune to the effects of malingering. Unfortunately, Subject 2, although he came from the sample of college "normals," was identified as being mildly organic when the criteria in the appendices were applied. Thus, in the case of Subject 2, we can merely conclude that it is possible for an organic $S$ to make himself appear to be much worse than he is by at least some of Canter's criteria. Perhaps the best evidence. for the second explanation is provided by an analysis of the test scores of the $11 \mathrm{Ss}$ judged to be not organic. The median difference score (i.e., "malingering 


\section{TABLE IV}

CANTER SCORES FOR COLLEGE STUDENTS UNDER STANDARD INSTRUCTIONS AND MALINGERING INSTRUCTIONS

\begin{tabular}{|c|c|c|c|c|}
\hline & St & $\begin{array}{c}\text { Scores } \\
\text { Under } \\
\text { Standard } \\
\text { Instructions } \\
\end{array}$ & \begin{tabular}{c}
\multicolumn{1}{c}{ Scores } \\
Under \\
Malingering \\
Instructions \\
\end{tabular} & $\begin{array}{c}\text { Difference Score: } \\
\text { Malingering Score } \\
\text { Minus } \\
\text { Standard Score } \\
\end{array}$ \\
\hline \multirow{3}{*}{$\begin{array}{l}\text { Diagnosed } \\
\text { as Having } \\
\text { Mild } \\
\text { Organic } \\
\text { Damage }\end{array}$} & 1 & 10 & 12 & +2 \\
\hline & 2 & 11 & 126 & +115 \\
\hline & 3 & 10 & 6 & -4 \\
\hline \multirow{8}{*}{$\begin{array}{l}\text { Diagnosed } \\
\text { as Being } \\
\text { Borderline } \\
\text { Organics }\end{array}$} & 4 & 6 & 9 & +3 \\
\hline & 5 & 3 & 72 & +69 \\
\hline & 6 & 9 & 35 & +26 \\
\hline & 7 & 13 & 20 & +7 \\
\hline & 8 & 0 & 20 & +20 \\
\hline & 9 & 0 & 56 & +56 \\
\hline & 10 & 7 & 50 & +43 \\
\hline & 11 & 8 & 22 & +14 \\
\hline \multirow{7}{*}{$\begin{array}{l}\text { Diagnosed } \\
\text { as Being } \\
\text { Not } \\
\text { Organic }\end{array}$} & 12 & 3 & 32 & +29 \\
\hline & 13 & 2 & 45 & +43 \\
\hline & 14 & 0 & 11 & +11 \\
\hline & 15 & 0 & 18 & +18 \\
\hline & 16 & 6 & 0 & -6 \\
\hline & 17 & 2 & 30 & +28 \\
\hline & 18 & 6 & 34 & +28 \\
\hline
\end{tabular}


score" minus "normal score") is approximately 25. Of the 11 difference scores, 10 are positive, and only 1 is negative. Using a sign test and testing an $\mathrm{H}_{0}$ of "no difference," one finds a $Z$ value of 2.39 , significant at $p<.05$ for a 2-tailed test. Testing $\mathrm{H}_{\mathrm{a}}$ : the difference is 210 with the same test, one finds a $Z$ value of 2.39 , significant at $\mathrm{p}<.01$ for a 1-tailed test. The choice of $\mathrm{H}_{\mathrm{a}}: \geq 10$ was significant. If a cutoff score of 9 is used with the Canter scoring system, then Ss who scored $\geq 10$ would be judged to be organic. Thus, even if a large group of normal Ss all scored 0 points under standard instructions by the Canter system, most of these Ss would still be expected to have difference scores $>10$ when they were instructed to malinger. Accordingly, if one used only the Canter system as a diagnostic tool, a large proportion of such Ss would probably be judged as organic. The refore, one can conclude, based on the data in Table IV, that Canter scores can be elevated substantially by most $S s$ when they are intent upon malingering.

If only the scores of "normal" Ss judged to be borderline or mildly organic are considered, no conclusive statements regarding the difference scores can be made due to the small numbers involved in both groups. However, it might be noted that the difference scores of these Ss showed a large amount of variability, specifically a range of -4 to +115. One might infer from the minus scores and from the low positive scores that some of these Ss experienced 
considerable difficulty in faking brain damage (i.e., drawing scorable distortions) under malingering instructions, but the reasons for this difficulty are not clear.

Given the weight of evidence from Table IV discussed above, one might question the validity of the Canter scores in identifying braindamaged Ss. But the problem is not that simple. Using a cutoff score of 9 , the scores did identify as normal all 11 Ss who were classed as not organic by the criteria used in the study without misidentifying any of the 18 organic Ss. Thus far, one could make an excellent case for the validity of the Canter scores in discriminating normals from organics. But under malingering instructions, only 1 of 11 Ss was correctly labeled as a malingerer. Thus, one can conclude that the Canter system will validly identify normal Ss under standard instructions, but that the scoring system will fail almost entirely when and if a normal $S$ wishes to feign brain damage.

The next problem is why the Canter system is not valid for malingerers. Canter's system includes many common distortions produced by organic Ss, but not all the distortions produced by them. Unfortunately, normal Ss can also produce many of these distortions when they are instructed to malinger, and thus their scores are usually indistinguishable from those of organics. The criteria used in this study, however, were more selective. They included only those distortions that organics commonly produce (some of these 
distortions are included in Canter's system but many are not) and malingerers rarely, if ever, produce. Also, the criteria in this study included negative weights, or malingering signs, which the clinician could use to identify malingerers. Canter's system did not include negative weights, apparently because he had not considered the problem of malingering. Thus, this study should be helpful to clinicians who either: 1) suspect that a patient is falsely claiming organic damage; 2) or suspect that a patient has been wrongly accused of malingering.

The Ability of the Pilot Study Criteria to Make Valid Differentiations

The fourth finding was that the criteria devised from the pilot study (see Appendices B, C and D) were sufficient to make valid sorting decisions, as evidenced by the results achieved in the main study. But perhaps more significant than the results themselves was the necessity of using a double-check (see "Note," Appendix C). The reason for the double-check is that organicity seems to lie on a continuum when assessed by the Bender. That is, many so-called normal Ss may evidence weak signs of brain damage whether given standard or malingering instructions, an indication that some normal Ss may have a negligible amount of brain damage which still places them within the range of normal functioning (note also the parallel argument raised before, that organics vary in the degree of their distortions). Therefore, 
a liberal interpretation of the criteria will result in a "no decision" verdict in a few cases; that is, the same Ss will be classified as malingerer and organic by the criteria. But the clinician should usually be able to reach a decision from the test information even if the messages he receives from the designs disagree. The question next becomes: How many organic signs were observed on the test record? A generous number increases the clinician's confidence that the record is organic. He then asks: How strong are the signs? Are the signs grossly distorted in a manner characteristic of Ss who are at least mildly organic, or do the signs seem to indicate a $S$ who falls within the range of normal functioning? The results from this study indicate that any combination of 3 signs is sufficient to warrant a diagnosis of at least mild organic damage. If the number and strength of the signs so indicate, the damage may be diagnosed as more severe. But one should properly ask: If the malingering criteria are valid, why should an organic $S$ be labeled as a malingerer? There are several possible answers.

First, the malingering criteria were chosen to describe distortions or techniques that were rarely found in the records of organics but were commonly found in the records of malingerers. If all the signs in a record point to malingering, then the probability of an $S$ 's actually being organic is negligible. However, it is possible that an otherwise organic record may include one or possibly two 
"malingering" signs without malingering having taken place. Given a sufficiently large sample of organics, rare signs will appear.

Second, the organic S may wish to look even worse than he is if the reward, or his needs, so dictates. Thus, the test picture would appear confusing, but for understandable reasons. In such a case, a retest several days later might produce inconsistencies which would tend to confirm the hypothesis that the $S$ is attempting to appear worse than he actually is.

Third, the S might be grossly damaged, in which case the criteria describing organic behavior on the test no longer apply. Bizarre scribbles is a case in point. Fortunately, gross damage of this type can almost always be verified by a thorough medical examination.

Fourth, the $S$ may be subject to psychotic episodes which result in test distortions similar to those produced by some malingerers. But a psychotic involvement can usually be diagnosed by the appropriate psychological tests.

In summary, what the fourth finding shows is that valid sorting decisions were made in about 908, or more, of the sorts without sophisticated diagnostic techniques. But certainly, questionable cases should be, and would be, investigated further when the clinician is confronted with an important decision of this type. 


\section{MAIN STUDY}

Since the purpose of the main study was to replicate the results obtained in the pilot study, few comments are necessary since that goal was achieved. But a few remarks are indicated.

First, the clinician who was not familiar with the pilot study (clinician 2) had to be trained to sort malingerers from organics. The criteria were first explained and discussed; then the clinician studied the protocols from the pilot study before he performed the sorts in the main study. Assuming that a clinician has had a reasonable amount of experience testing organic Ss with the Bender-Gestalt, the training should not require more than about one hour in time.

Second, it was decided that an unequal number of organics and malingerers should be used in the main study to make the sorting procedure more difficult. Previously, clinician 1 had expected a more or less equal division between the two groups, and thus he was provided with a clue which he normally would not have had in actual clinical practice. Since one would expect organics to outweigh malingerers in practice, it was decided that the organic group should exceed the malingerer group in size. The clinicians were told only to expect some malingerers and some organics, but the exact number of cases in each group was not revealed.

Third, the missorts of clinician 1, who had participated in the project from the beginning, were most instructive. 
As noted previously, 5 of the 33 organics were misclassified as malingerers. Of these 5 misgrouped organics, 2 were so extensively damaged that their records could best be described as nearly incoherent scribbles. One similar organic "scribble record" was correctly grouped, but the clinician expressed uncertainty in all 3 cases and stated that a positive diagnosis could only be made if he had access to the time the Ss needed to complete the test (his assumption was that extensively damaged organics would probably require considerably more time to complete the test sequence than malingerers who pursued a scribbling strategy). One might speculate at this point that test time and a Wechsler IQ testing would probably be sufficient to eliminate nearly all missorts. The Wechsler test results should provide a means of checking the diagnostic hypothesis derived from the Bender record. For example, badly damaged organics usually achieve low Wechsler IQ scores, and the subtest scores usualiy fall in some rational pattern. On the other hand, one would expect organics who were able to achieve post-trauma Wechsler scores in the superior or very superior range, for example, usually to produce Bender records of superior quality relative to organics of average or less than average post-trauma Wechsler performance. However, from a practical standpoint, missort errors with extensively damaged organics in this study are not really critical because in the real world the clinician would have more than ample evidence (medical and 
otherwise) of organic damage for a court case. From the results of this study, one could infer that clinicians would require more information than the Bender record in order to decide whether a record consisting of scribbles came from an extensively damaged organic or from a malingerer. Regarding the 3 remaining missorted organic records from the initial 5 , these records could be described as follows: one contained extremely bizarre distortions for an organic with traumatic brain damage (i.e., signs of other kinds of disturbance were primary, although organic signs of secondary importance were also apparent); one contained figures which could only be interpreted as indicating no organic damage, while other figures pointed toward a diagnosis of extensive organic damage (i.e., the record was grossly inconsistent, usually a strong sign of malingering); one contained only a few weak organic signs and thus appeared to be borderline at worst (this $S$ had a Wechsler IQ in the superior range despite a severe head injury). In practice, only the last record described would present any problem in a hypothetical court case. The problem here would not be to determine whether organic damage had occurred since medical evidence was readily available, but rather, to determine the degree of damage sustained. The most practical implication of the main study is consistent with a conclusion derived from the pilot study: test records pointing toward mild organic damage present the most difficult sorting problem. Records 
with bizarre distortions or scribbles present few problems in the real world since other information, such as test time, Wechsler scores, medical evidence and interview data would be available.

Fourth, malingerers were easily identified relative to organics, since their test taking strategies revealed patterns which usually differed markedly from those of organic Ss. The Ss who presented the greatest sorting challenge were the 4 "normals" in Sort 2 who had apparently sustained some type of central nervous system disturbance and thus were indistinguishable from borderline or mild organic ss in the organic group. But the reasons for this high proportion of what seemed to be marginally organic Ss in a population of college Ss are not clear (drug involvement is only one previously mentioned possibility) and should be investigated. One point is clear, however. When Ss radically change their normal test taking strategy in order to appear braindamaged, they can be identified as malingerers. A malingering strategy appears to be successful only when "normals" who indicate CNS disturbances during baseline testing maintain their essential test-taking behavior under a malingering set. ${ }^{4}$

${ }^{4}$ This hypothesis is supported by the previously discussed pilot study results and by the results of the main study. In the main study $3 \mathrm{Ss}$ were identified as being mild organics according to the pilot study criteria. These records were coded and included in both sorts for the purpose of testing this hypothesis with the understanding that the results of both sorts would not be included as part of the data in Table $I$. One of these Ss deviated only slightly from his baseline performance under nalingering instructions and hence was identified as borderiine organic. The other 2 Ss adopted transparent malingering strategies and were easily grouped with the malingerers. Al1 3 ss were grouped with the organics in the normal-organic sort. 
In other words, it is extremely unlikely that a normal $S$ could "fake" brain damage unless he had a CNS disturbance in the first place and essentially 'played it straight.'

Fifth, the sorting performance of clinician 2 (who had little recent experience with the Bender records of organic Ss) was almost as good as that of clinician 1 (the "expert"). This finding indicates that clinicians can be trained to use the criteria detailed in the study with considerable validity and reliability.

The Questionnaires

Some comments about the questionnaires used in the study are also in order. The questionnaires were to serve three major purposes: 1) to identify as probable organics those "normal" Ss who were not screened out during the Bender test administered under standard instructions; 2) to provide additional confirmation of brain damage in Ss who had been judged organic in the initial screening; and 3) to discover whether the re was any connection between what a $S$ knew about the Bender test and his success as a malingerer. In general, the questionnaires did not prove particularly helpful for several reasons. First, brain damage can be caused by many factors concerning which the typical $S$ would have little, if any, information (e.g., birth trauna and pre-natal maternal illness). Second, the $S$ could have forgotten significant events, such as high fevers, or perhaps he considered them unworthy of mention. 
Third, he might not wish to admit that he had brain damage, assuming that he knew. Fourth, if he was a heavy user of drugs, the $S$ might not trust the $E$ with such information even if he realized that he might have sustained brain damage by such means. Fifth, so few Ss were able to malinger successfully that no conclusions regarding malingering and knowledge of the test could be drawn.

\section{Further Studies}

The experiment also raised some pertinent questions which fell beyond the scope of this study. Two of the more important issues dealt with the topic of drugs. First, would Ss who were under the influence of halucinogenic drugs at the time of testing malinger more convincingly than Ss who were not? Obviously, such a question (and any question dealing with illegal use of drugs by human Ss) creates some thorny ethical and legal problems, but the question would have some considerable pragmatic importance if we could assume for the moment that a $S$ under an altered state of consciousness might perform on the Bender in a manner similar to an organic S. Second, do Ss who have extensively experienced halucinogenic drugs have a higher incidence of brain damage than a comparable sample of Ss who have not? If so, assuming some kind of causal relationship, the kind of organic damage might form some type of characteristic pattern on the Bender distinct from that caused by traumatic injury and other kinds of organic damage. The second 
question is especially pertinent to the topic, "Limitations of the Study," point 4, discussed below.

Limitations of the Study

Obviously, an exploratory study of this type will have its limitations. Five of the more important ones are detailed below:

1) It was not possible for financial reasons to substantiate by medical and physiological evidence our contention that some of the "normal" Ss in the experiment had sus tained brain damage. Such proof would further strengthen the argument that the criteria validly identify Ss with brain damage.

2) Generally, the criteria used in the study comprise a qualitative approach, although some quantitative techniques were used. Certainly, a more nearly quantitative approach would be desirable to facilitate training in the system. Further, the clinician who wishes to use this system in his practice is strongly advised to gather a sample of malingering normals for self-training purposes. One of the primary reasons for collecting such a sample is that in this system, just as in the Canter system, some distortions are classed as organic and some are regarded as being within the normal range of functioning, depending upon the degree of distortion. In general, we assumed that a distortion met the criteria even if the distortion was very slight, slight that is 
relative to the scoring criteria detailed in the Canter system. But the reason for our relatively bold approach to scoring deviations was our confidence in the negative weights which were used. Thus, if both organic and malingerer signs were scored, we exanined the degree of the distortions scored. Accordingly, what was lost in a standard measurement approach was offset by a highly flexible diagnostic procedure with built-in checks and bàlances.

3) Strictly speaking, the criteria used in the study should be considered valid only for organics with traumatic damage caused by an external blow to the skull--as opposed to stroke victims, epileptics, etc.--even though it is extremely doubtful that the criteria for malingerers would vary much if similar research was to be pursued with other organic groups. However, the criteria for specific kinds of organic damage (e.g., epileptics) almost certainly would change. Also, the results of the study would generalize, strictly speaking, only to Ss of at least normal intelligence with a college background and an age range of about 20-40 years. Although there is little reason to believe that age, intelligence or education are variables which are relevant to the simulation of brain damage, the possibility exists and should be investigated through an appropriate research design. 
4) The test would probably identify as organic any $S$ who had in his past sustained significant brain damage, assuming that the effects of the damage were still evident at the time of testing and assuming that the $S$ did not adopt a malingering strategy and attempt to look much worse than he actually was. Thus, the criteria probably would not be able to help the clinician determine whether a specific incident did or did not cause the organic damage inferred from the distortions on the test record. Specifically, let us assume that a $S$ 's record is determined to be organic and that this $S$ was involved recently in an automobile accident. The clinician cannot assume that the organic damage inferred from the test record was caused by the car accident. He must still demonstrate a causal relationship between the two events. The use of the criteria in this study merely allows the clinician to explore the issue of malingering with a scientifically validated method.

5) The procedure used in the main study should be replicated with a new sample of organics and malingerers. Since the criteria devised in the pilot study were applied retrospectively to the $S s$ in the two sorting groups, the main study would not be considered a replication of the pilot study results according to generally accepted scientific procedures. 


\section{SELECTED REFERENCES}

Bender, L. A visual-motor Gestalt test and its clinical use. New York: The American Orthopsychiatric Association, 1938.

Billingslea, F.Y. The Bender-Gestalt: An objective scoring method and validating data. Journal of Clinical Psychology, 1948, 4, 1-27.

Blum, R.H. E Nims, J. Two clinical uses of the Bender visual-motor Gestalt test. U.S. Armed Forces Medical Journal, 1953, 4, 1592-1599.

Canter, A. The background interference procedure for the Bender-Gestalt test. Preliminary manual of scoring an interpretation. University of Iowa, 1966. (Mimeo)

Canter, A. BIP Bender test for the detection of organic brain disorder: Modified scoring method and replication. Journal of Consulting and Clinical Psychology, 1968, 32, $522-526$.

Hain, J.D. The Bender-Gestalt test: A scoring method for identifying brain damage. Journal of Consulting and Clinical Psychology, 1964, 28(1), 34 .

Hays, W.L. Statistics. New York: Holt, Rinehart and Winston, 1963.

Pascal, G.R. \& Sutte11, B.J. The Bender-Gestalt test: Its quantification and validity for adults. New York: Grune \& Stratton, 1951.

Tolor, A. The graphomotor techniques. Journal of Projective Techniques, $1968,32,222-228$.

Wagner, E.E. \& Murray, A.Y. Bender-Gestalts of organic children: Accuracy of clinical judgment. Journal of Projective Techniques Eq Personality Assessment, 1969, $33(3), 240-242$. 
SAY I VNNOILSBNO

$\forall$ XIANadd 
Questionnaire I

Name :

What do you think was the purpose of this experiment? 
Name :

Check the appropriate items if you have experienced any of the following:

Scarlet fever, meningitis, encephalitis

Sustained fevers which were high enough to cause delirium, seizures, or spasms.

Head injuries serious enough to cause unconsciousness or hospitalization.

If you have checked any of the above, please explain.

Do you have any reason to suspect that you may have sustained brain damage?

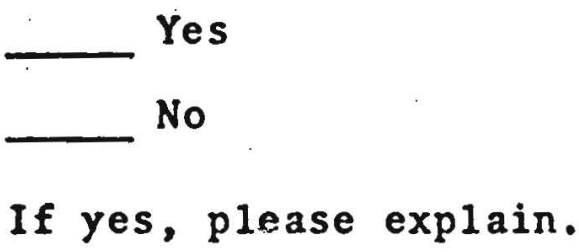


Questionnaire III

Name :

The test with the nine designs which you took is called the Bender-Gestalt. Please tell me what you know about this test.

In your opinion, what kinds of mistakes would a person with brain damage make who took the test? 
VIYGLIYD JILSONDVIA THILNGYGHAI

g XIđNGddV 
Differential Diagnostic Criteria: Organics vs Malingerers

1. Organics generally simplify the drawings; they rarely complicate them.

a. Gross, bizarre or complex distortions are characteristic of malingerers, not organics.

2. Any component element (e.g., line, circle, dot, curve or angle) which is markedly distorted in one design by an organic $S$ is likely to be similarly distorted in other designs with the same element.

a. If circles, dashes or other distortions occur in Design 1 , it is likely that similar distortions will occur in Designs 3 and 5 if the $S$ is organic. However, different organic distortions will occasionally occur. Gross inconsistencies are a sign of malingering.

i. Common organic distortions are dots rendered as follows: 6's--either vertical, or rotated $90^{\circ}$ counterclockwise; 2 's, or circles with one or two "tails"; dashes and/or double connected dashes (which may appear as u's or h's); "bumblebee" dots; "clod" (large and irregular) dots; circles only partially filled.

ii. If the organic $S$ experiences difficulty in rendering the dot "angles" in Design 3, he should experience similar difficulty with the angle of the extension in Design 5. Marked 
discrepancies of quality in rendering the gestalten of these two designs is a sign of malingering.

b. Regarding Design 4, most organics experience considerable difficulty in rendering the shape of the curve accurately, particularly the ends, which are often discrepant in at least minor ways; however, malingerers often grossly distort the curve or draw it too well. Further, organics tend to draw curves in Designs 4 and 6 which are similar in quality and distortions; a gross difference in curve shape and quality on these designs is a sign of malingering.

i. The organic $S$ tends either to miscenter the curve of Design 4 by a slight amount, whether the two figures on his reproduction touch or not, or to move the curve up slightly so it appears noticeably up the base of the open square. But gross miscentering distortions with bizarre figural rotations and/or substantial (more than $1 / 3$ of the curve height) figural overlaps, are rarely produced by organic Ss.

ii. The organic $S$ may distort the angles of the open square on Design 4, but only rarely will he round an angle or render the open square as a curve; the latter variety of distortions 
are usually peculiar to malingerers.

c. Organic Ss commonly produce the following distortions on Designs 7 and 8: "dog-eared" angles on the intersection of two lines; non-connected lines; often with a gap of $1 / 16$ inch or less but sometimes greater; one or two line extensions (often relatively slight) at the intersection of any two lines; "lost" or distorted angles, usually produced by varying the length of the short lines at the ends of the hexagons; extra angles often produced by changing the direction of a line so as to cause it to join another line in the design. Organic Ss may also "lose," or nearly lose, an angle in these designs, but they rarely, if ever, round an angle so as to produce a "bullet-shaped" figure. The latter distortion is characteristic of malingerers. Further, many malingerers draw these figures much too regularly; that is, the lines are often smooth and straight, and it is apparent that the $S$ has never removed his pencil from the test paper once he began drawing the figure. Implied in such "regular" productions is a perceptual and/or motor ability which organics rarely have.

3. Organics tend to be consistent in the level of their figural distortions; that is, if a simple design, such as Design 1, is seriously distorted, it is unlikely that a relatively more complex design, such as Design 3, 
would be drawn more accurately in relative terms.

4. Organics produce certain pathognomonic, unique responses. When these responses appear in records conforming to the criteria of organicity listed above, these records can be classified as organic.

a. Most organic records will show a very slight to moderate bow, wave or ripple effect on Design 2 . In contrast, malingerers will either produce a much too regular (linear) rhomboid quadrilateral (in outline) or produce bizarre shape distortions in the gestalt. These distortions in effect suggest a completely different figure from the original.

b. Characteristically, organics with moderate damage will produce several figures which are rotated $10-45^{\circ}$ from the originals. Organics with more extensive damage may rotate at least one figure as much as $90^{\circ}$. However, $180^{\circ}$. rotations rarely; if ever, occur in organic drawings; such rotations should be interpreted as a sign of malingering.

c. Mild to moderate tremors (i.e., shakey, unsure lines) are frequent in most organic records. !lowever, gross, exaggerated or bizarre tremors are characteristic of malingering.

d. On Designs 3 and 5 , organics commonly produce the distortions described under 2a. But these dot designs are rarely, if ever, rendered as lines. 
Such distortions are usually peculiar to malingerers.

e. Organics commonly experience difficulty with the intersection of the figures in Design 6. Regardless of the kind of record, malingerers seldom demonstrate this difficulty. Usually, one or more of the following occur on organic records: there is a gap, often small, in one of the curved lines near the intersection; the sinusoidal curve on the "vertical" sequence is elongated and/or noticeably flattened at the point of intersection with the horizontal curve sequence; a straight, or nearly straight line is drawn in the "vertical" curve sequence near the point of intersection. i. Organic records often show one or more second attempts (as described by Canter, 1966) in portions of Design 6. The protocols of malingerers rarely reveal second attempts (this principle holds for other designs also, but is less likely to be true of Designs 7 and 8). Malingerers commonly draw Designs 6 and 8 with regularity and seeming assurance (few lines indicate uncertainty or hesitation) despite the odd distortions which may appear in their figures.

ii. Organics commonly peak, square or flatten the sinusoidal curve in Design 6 . The curves 
which malingerers produce are much less consistently distorted.

iii. Some organics will bow the "vertical" curve sequence slightly in Design 6 . Generally, the "vertical" sinusoidal curves are more variable in size and quality of reproduction than those in the horizontal sequence. The protocols of: malingerers usually reveal that the sinusoidal curves are either strikingly similar to those in the test design (i.e., they are too accurate) or that they are distorted in a bizarre fashion.

f. As severe distortions (detailed previously) appear in more increasing severity in an organic record, the figural lines will commonly reflect hesitation and uncertainty: 1) slight tremors often appear; 2) lines in quadrilaterals and hexagonals are often not connected or are over-extended; 3) "dog-eared" angles often appear in quadrilaterals and hexagonals; 4) differential line pressures are often evident; 5) guide dots may be used to establish figural bounds; 6) lines in quadrilaterals and hexagonals may abruptly change direction (scored as extra angles by Canter, 1966). The records of malingerers with distortions of comparable severity are often drawn too regularly, with few signs of uncertainty or hesitation as described above. 
APPENDIX C

CHECKLIST FOR MALINGERING 
Checklist for Malingering

Classify as malingering if any one of the following appears:

1. If any figure is rotated approximately $180^{\circ}$

2. If any two angles in a quadrilateral or hexagonal are rounded.

3. If any hexagonal appears "bullet-like" in shape.

4. If the open square in Design 4 is rendered as a curve, or if any square or diamond is noticeably circular in shape.

5. If the dots in Designs 3 or 5 are rendered as lines.

6. If the dots in Design 1 are significantly distorted such that they are not recognizable, or barely recognizable, as a line and the essential gestalt of any other design is more or less preserved. The same principle applies to the gestalt of Design 2 .

7. If Design 6 shows no organic signs (see 4e, Appendix B) and no signs of hesitation or uncertainty (see $4 f$, numbers $i$ and $i i i$; Appendix B). If in doubt, do not classify.

8. If gross, exaggerated tremors occur.

9. If complex, highly bizarre distortions occur (exclude scribbles, which may occur in severely damaged patients).

10. If Design 2 is drawn in outline as a rhomboid quadrilateral with the top and bottom lines almot perfectly straight (no bows or bends). If the re is substantial doubt, do not classify. 
11. If the Canter score (1966, pp. 5-28) is 9 or less.

Note: If three or more organic signs, as described in Appendix $B$, occur when the $S$ has been classified as a malingerer; then at least three possibilities exist: 1) the individual is at least mildly organic, but he is also malingering (i.e., trying to look worse than he really is); 2) his organic impairment is gross (a possibility which could probably be verified medically); 3) he is also psychotic, or subject to psychotic episodes (this possibility could be investigated through the appropriate psychological tests). 


\section{APPENDIX D}

\section{CROSS CHECKS FOR DISTORTION CONSISTENCY}


Cross Checks for Distortion Consistency

As discussed in Appendix B, and elsewhere, organics generally produce distortions which are consistent between similar designs. Gross distortion inconsistencies are usually a sign of malingering.

1. Compare distortions of the dots on Designs 1,3 and 5 .

2. Compare angle distortions of dot rows on Design 3 with the distortions of the angle of the extension on Design 5. Severe distortions in Design 3 should result in at least a moderate distortion of the angle of, and/ or the placing of, the extension.

3. Compare distortions in the curve of Design 4 with the curve distortions in Design 6 . Generally, if the curve on Design 4 is distorted, Design 6 will be worse, but the distortions on Design 6 should be at least somewhat similar in kind.

4. Compare the angles made on the following designs:

1) the curve with the open square on Design 4 ; 2) the extension with the curve on Design $5 ; 3$ ) and the "vertical" sequence of curves with the horizontal sequence of curves on Design 6. Organics will generally do equally well or equally poorly at reproducing these angles. The same applies to the touchpoint or the point of intersection (as appropriate) on the three designs.

5. Compare the overall quality of designs 7 and 8 . 


\section{APPENDIX E}

\section{SAMPLE ORGANIC AND MALINGERER PROTOCOLS}


ORGANICS 
64<smiles>C1CCC2(C1)CCC2</smiles>

Vary mill organic divulge. I had wechsler ifS 1.9. of 105.

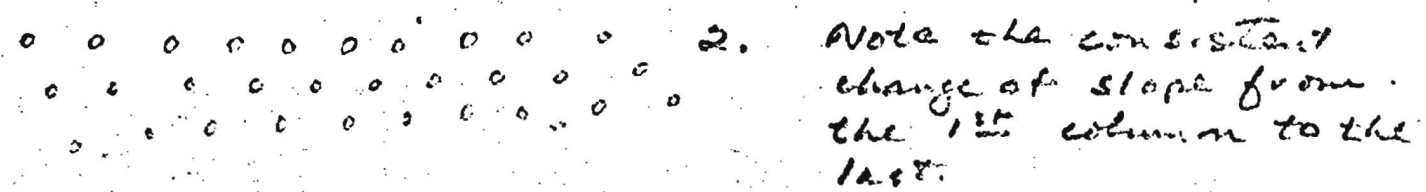

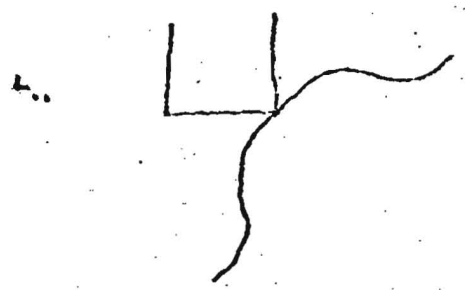

curve listortim.

Compare with Design 6

Excellent reproduction

for an organic record

5.

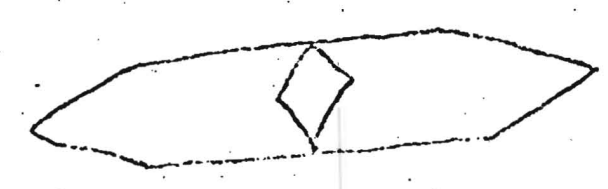

$\therefore$

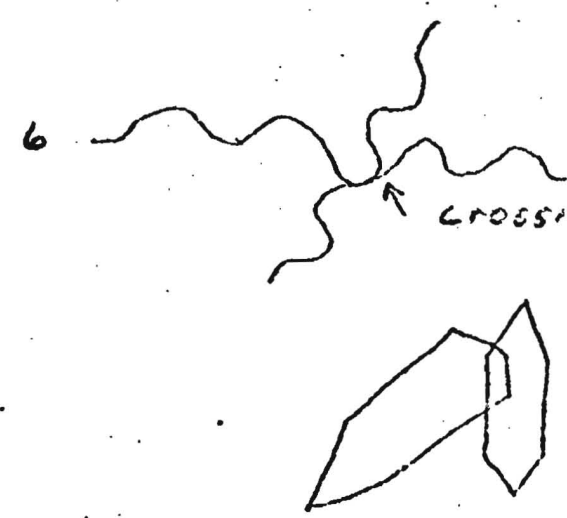

distortion ins size

between these two (figures are

figures. 


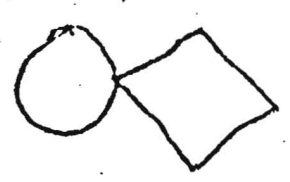

Llidol organie domage.

I extra columos

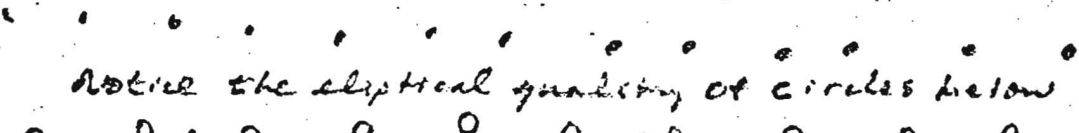

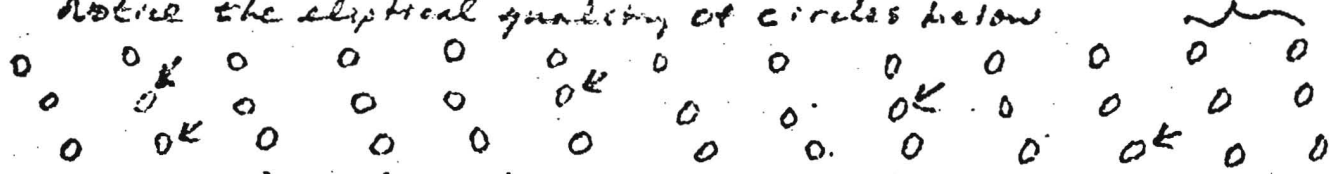

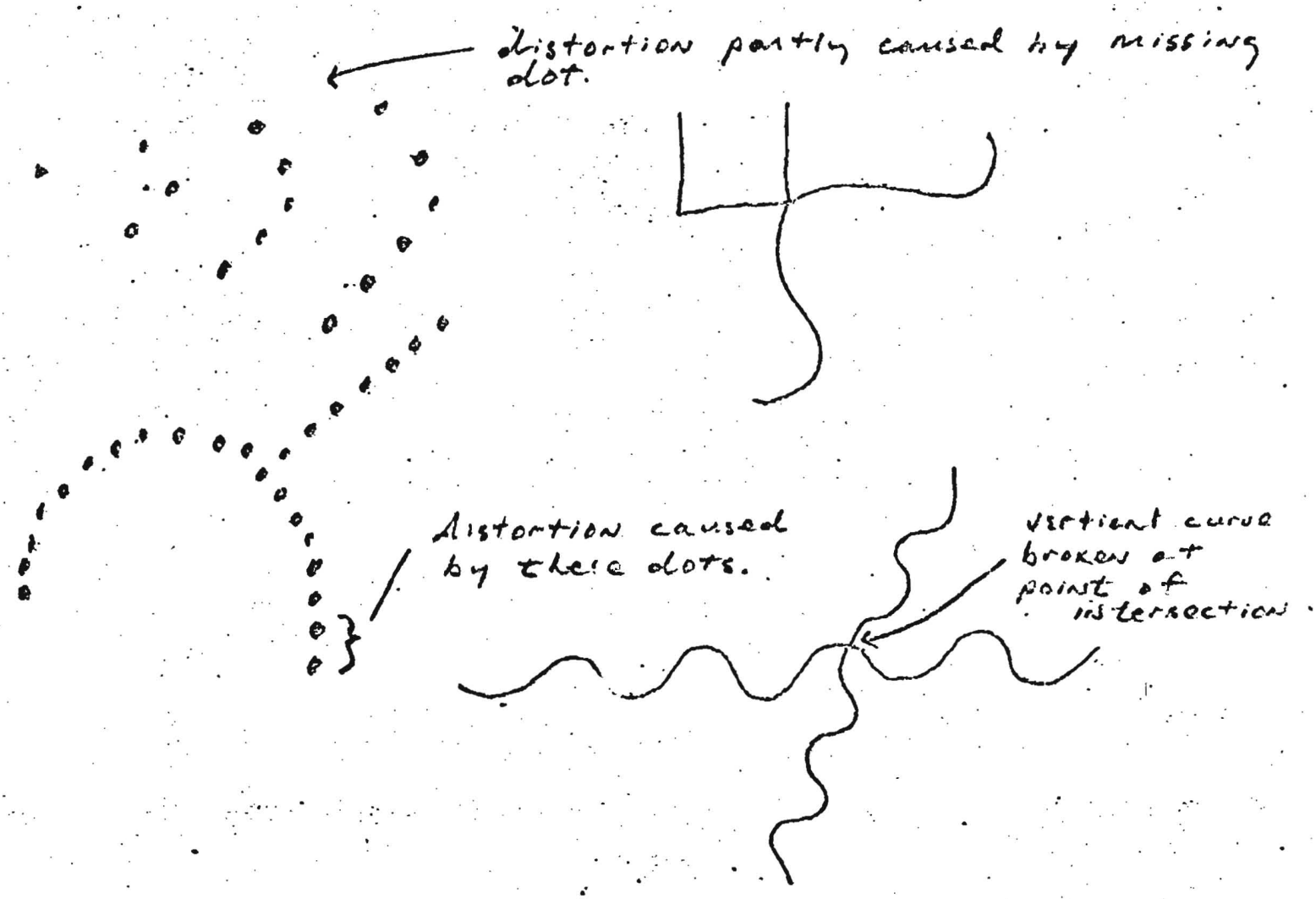

$: \quad 2$
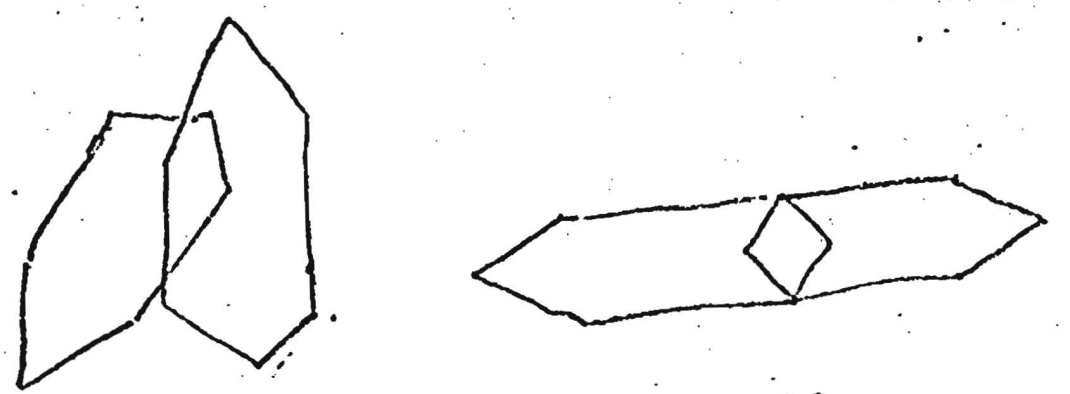

(figares slightly 

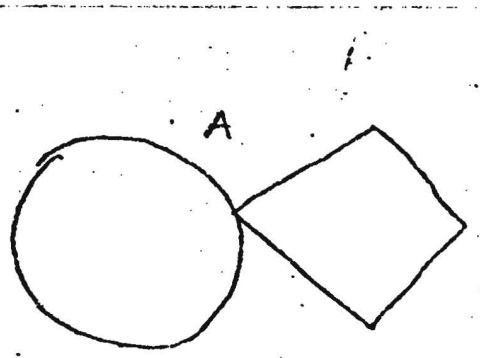

parting filled

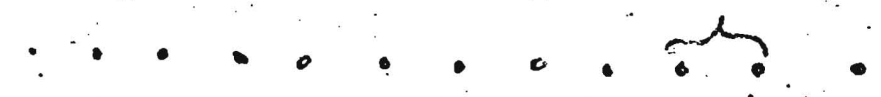

$\begin{array}{lllllllllll}0 & 0 & 0 & 0 & 0 & 0 & 0 & 0 & 0 & 0 & 0 \\ 0 & 0 & 0 & 0 & 0 & 0 & 0 & 0 & 0 & 0 & 0 \\ 0 & 0 & 0 & 0 & 0 & 0 & 0 & 0 & 0 & 0 & 0\end{array}$
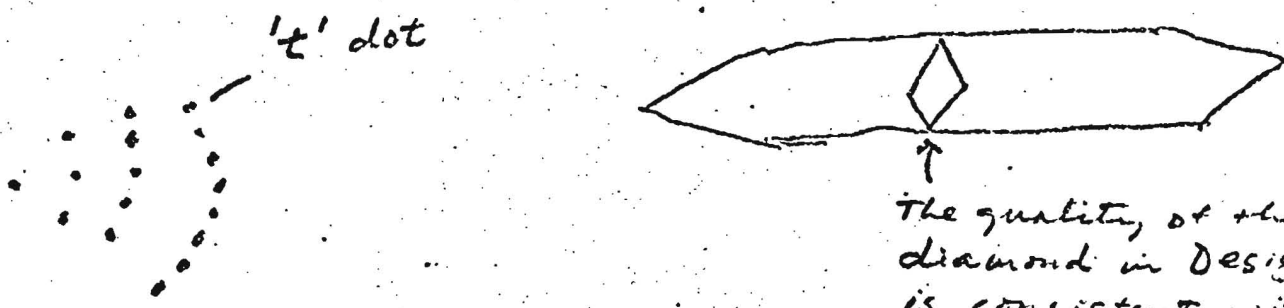

The guntity, of the

diacustal in Desigit $s$ is consistent with the qualtey ot tha

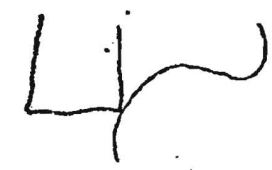

dianumet in $A$.

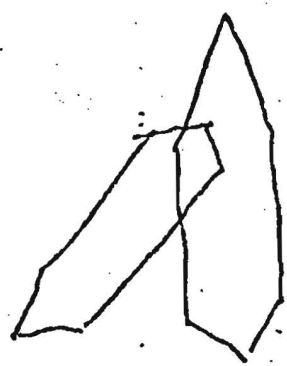

deartidnd in $A$.
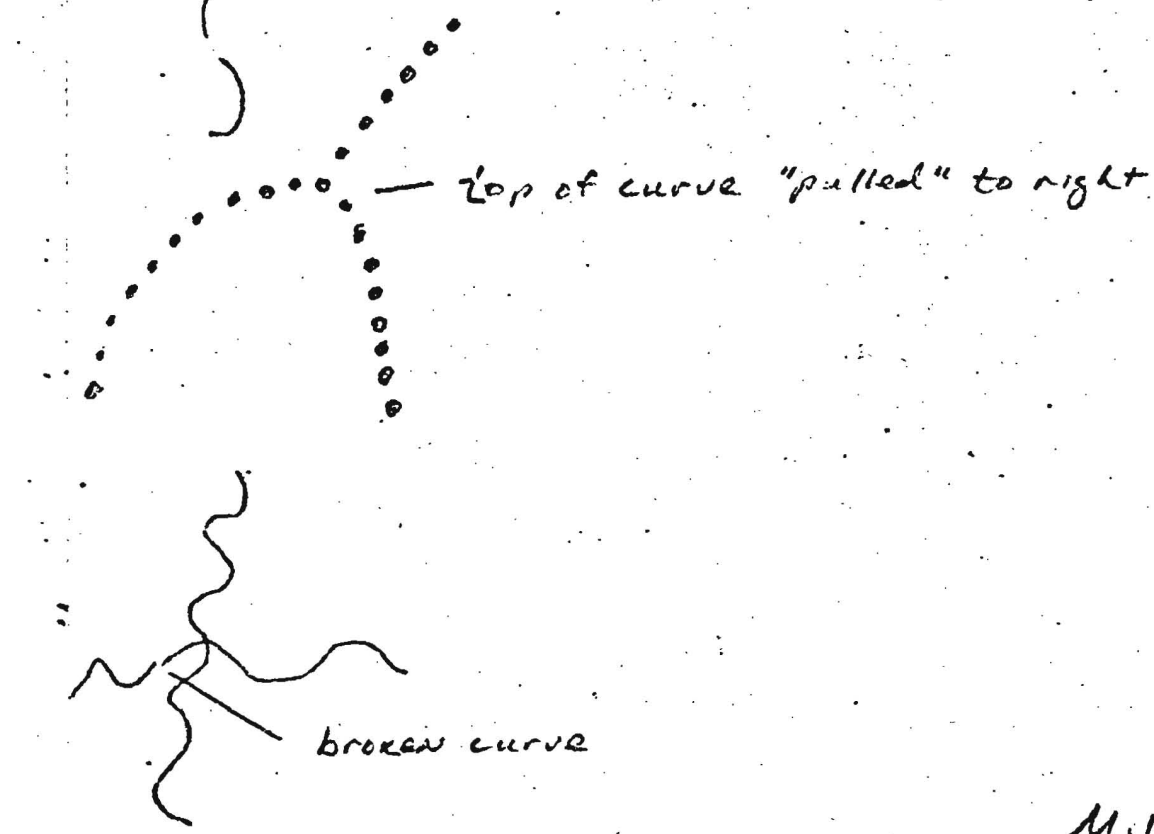

Mild organic drameries

(figures reduceat to, ti.) 
67
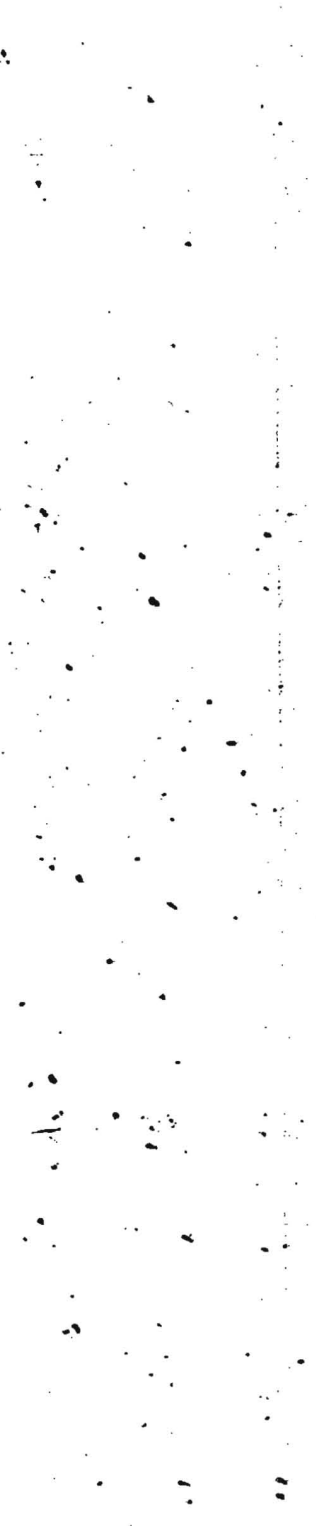
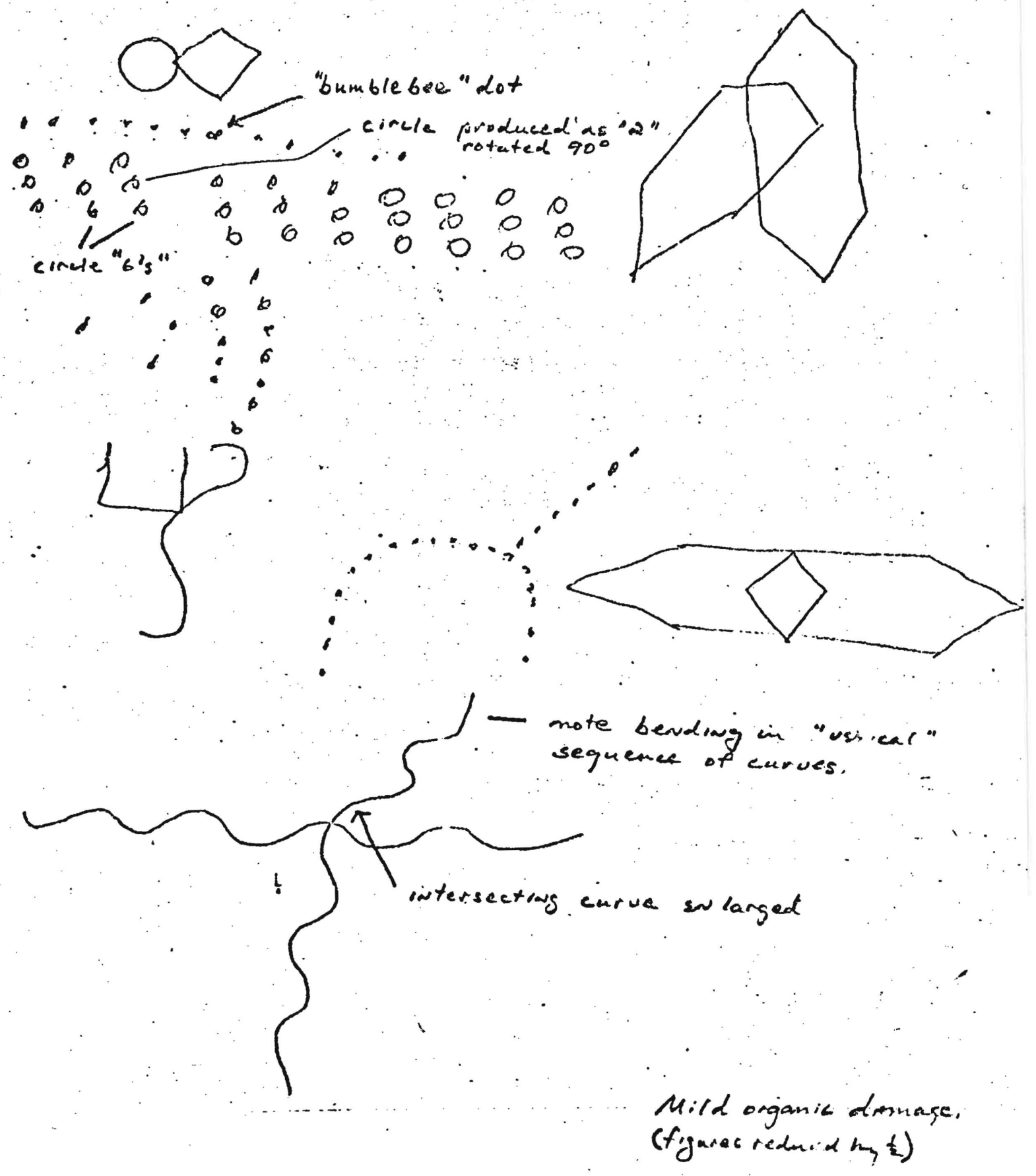


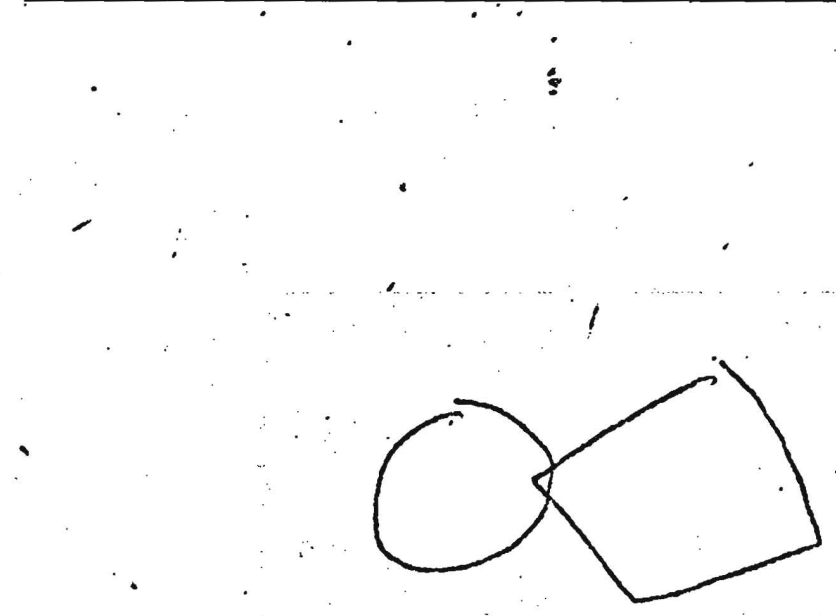

68

$\therefore \quad$ a dots poorly executed but love is regular.

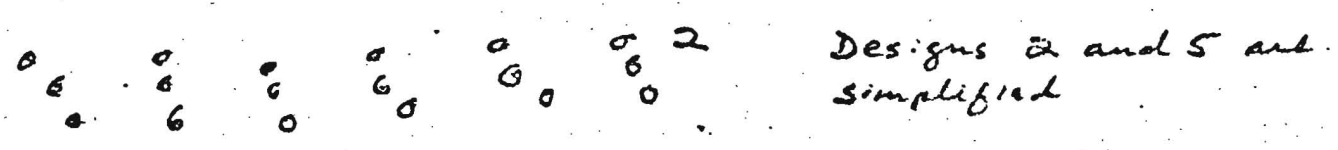
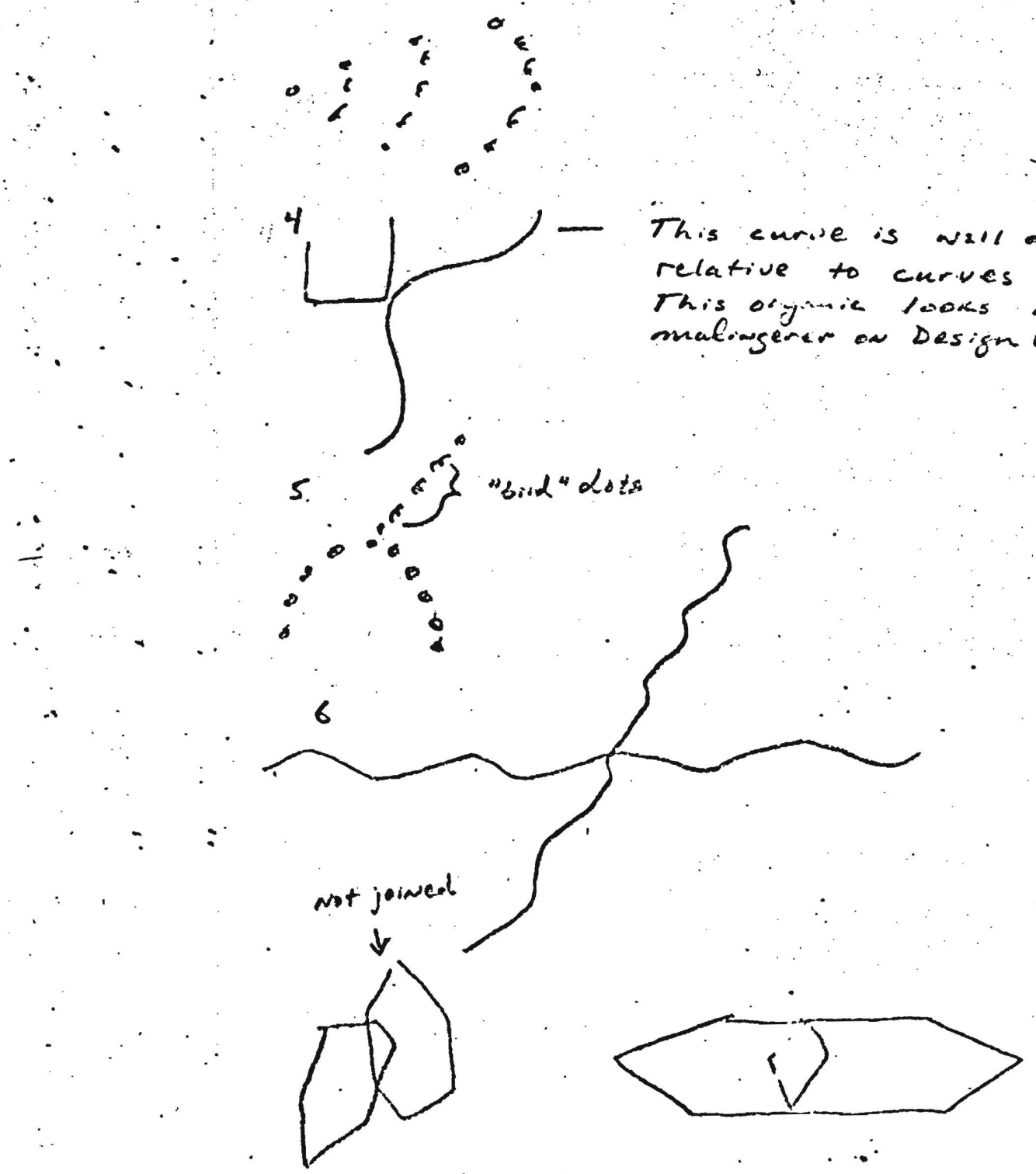


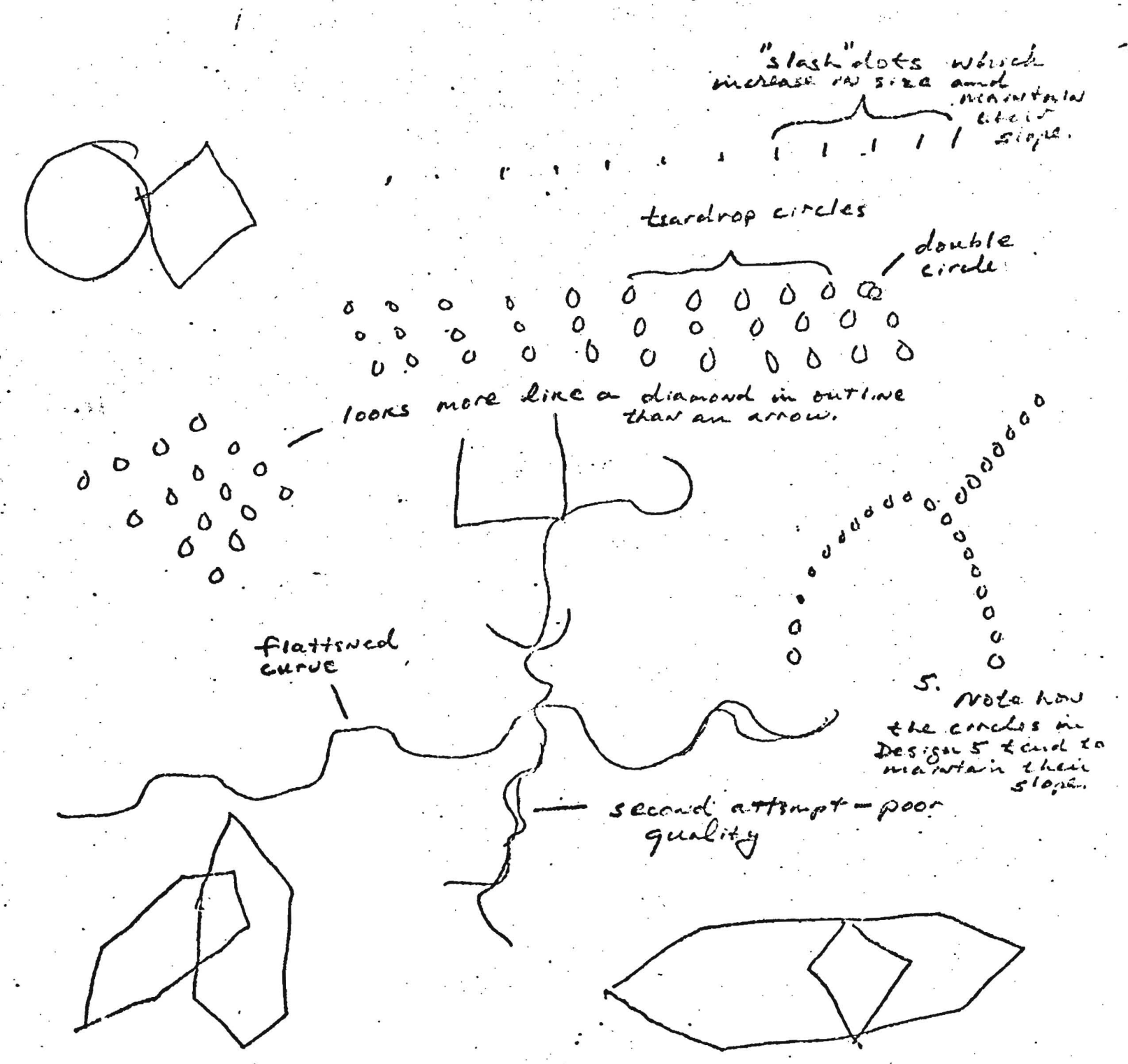

Moderate organie

(figures sechesed thy t) 


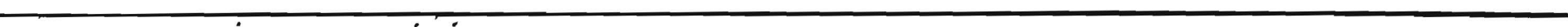

70
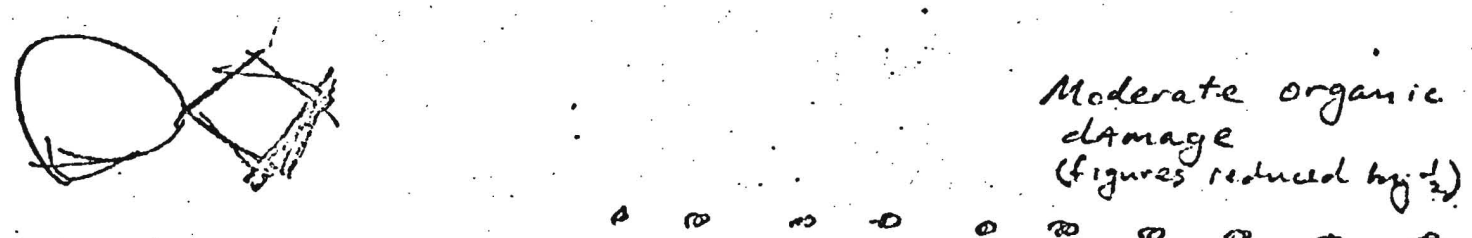

Moderate organie. clamage

3000

( $\infty \circ 0^{a}$ $\begin{array}{llllllllll}0 & 0 & 0 & 0 & 0\end{array}$ 6. 6

o. 0 6 8

$0 Q$ (3) 60

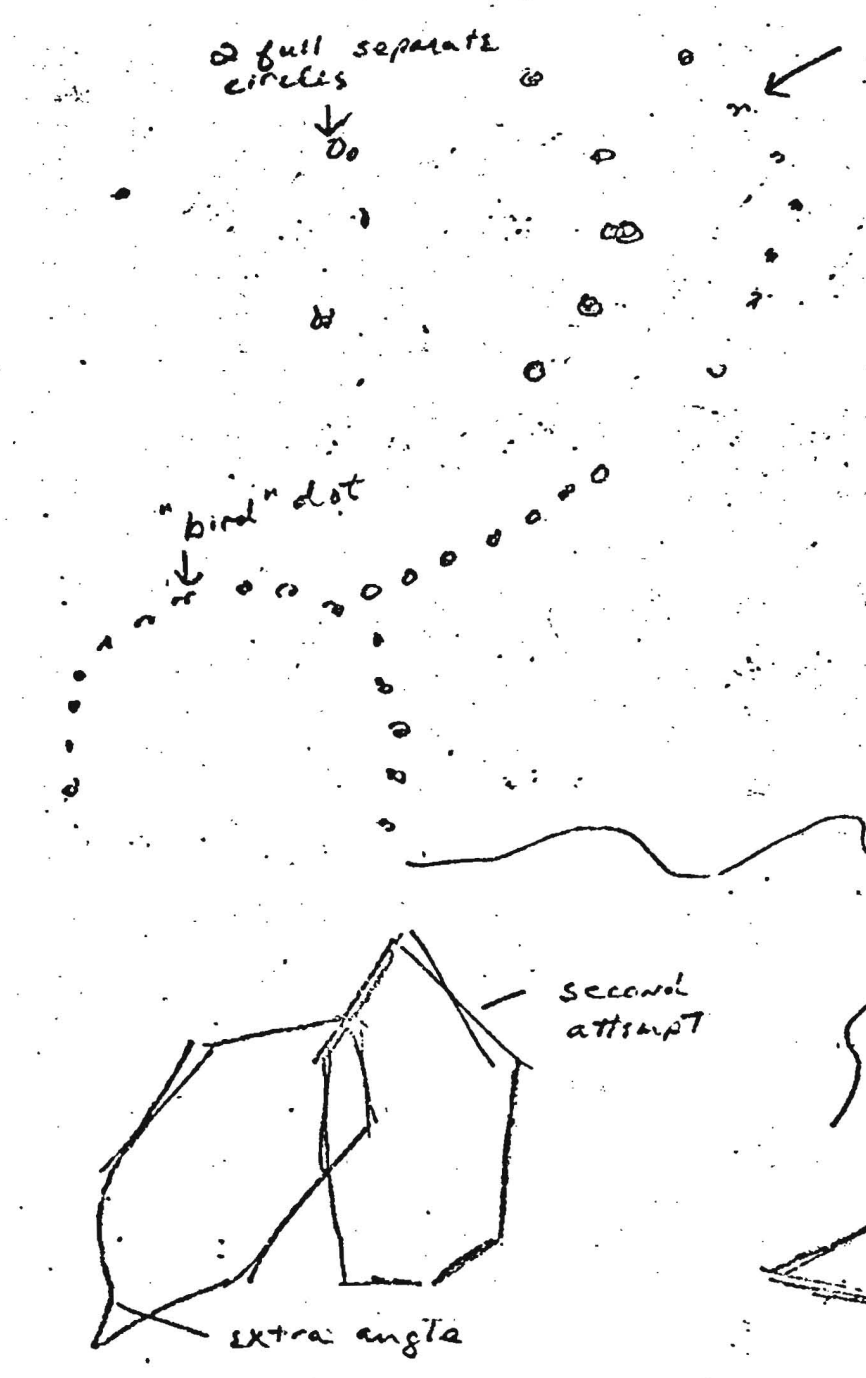

"bird" dot

(ब) $B$

D 6

$\because$ 


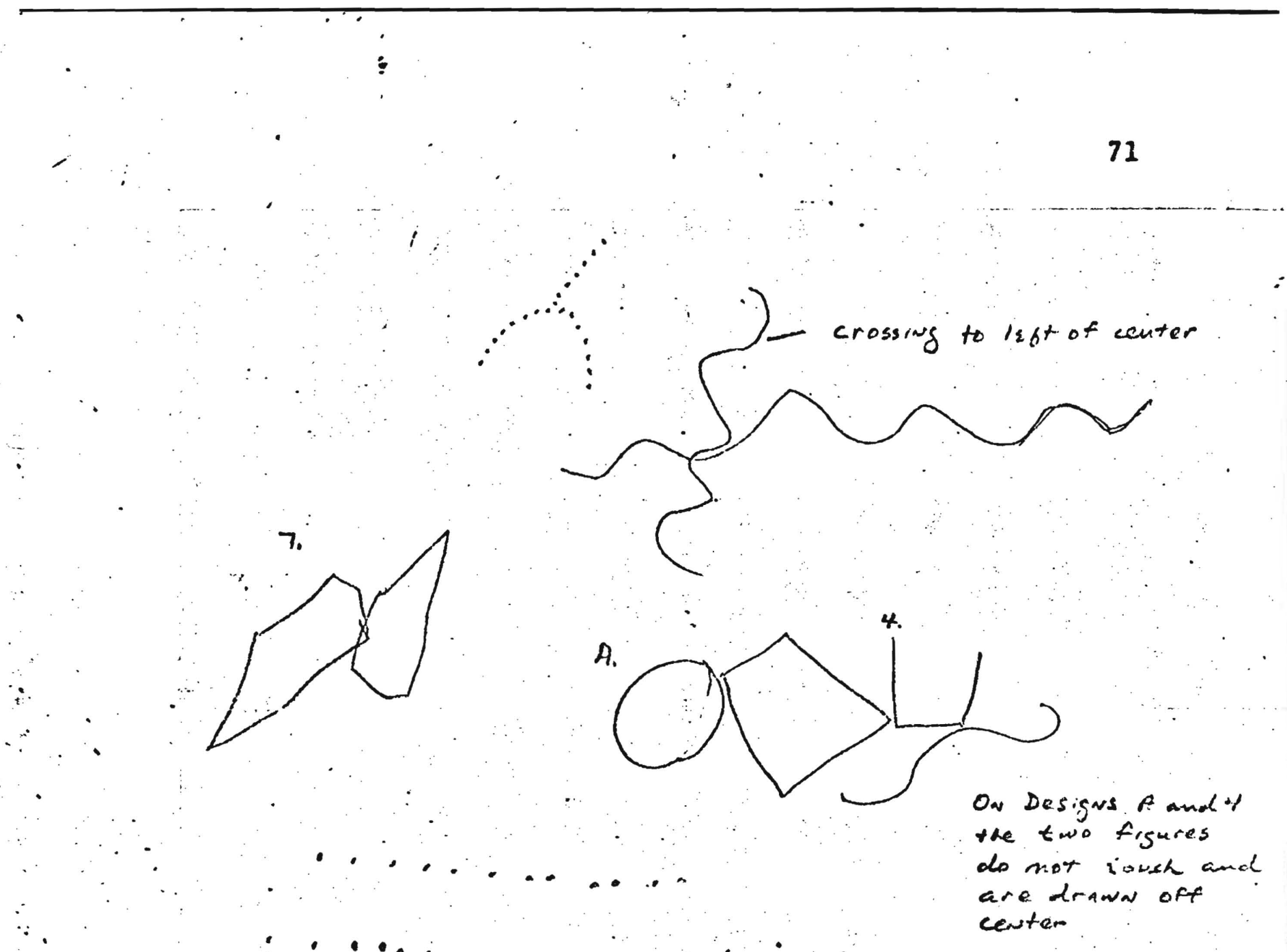

$\therefore \quad \therefore \quad$ Desigu 8 : poom reproduction. Tet nete overall $\because \quad \therefore \quad$ Similarity to Design 7.

8.

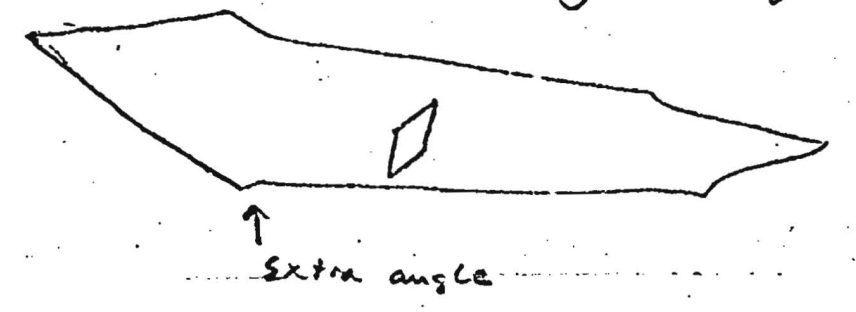

Moderatc orgianic.

drinage.

(figures resienceliby $\frac{1}{2}$ ) 


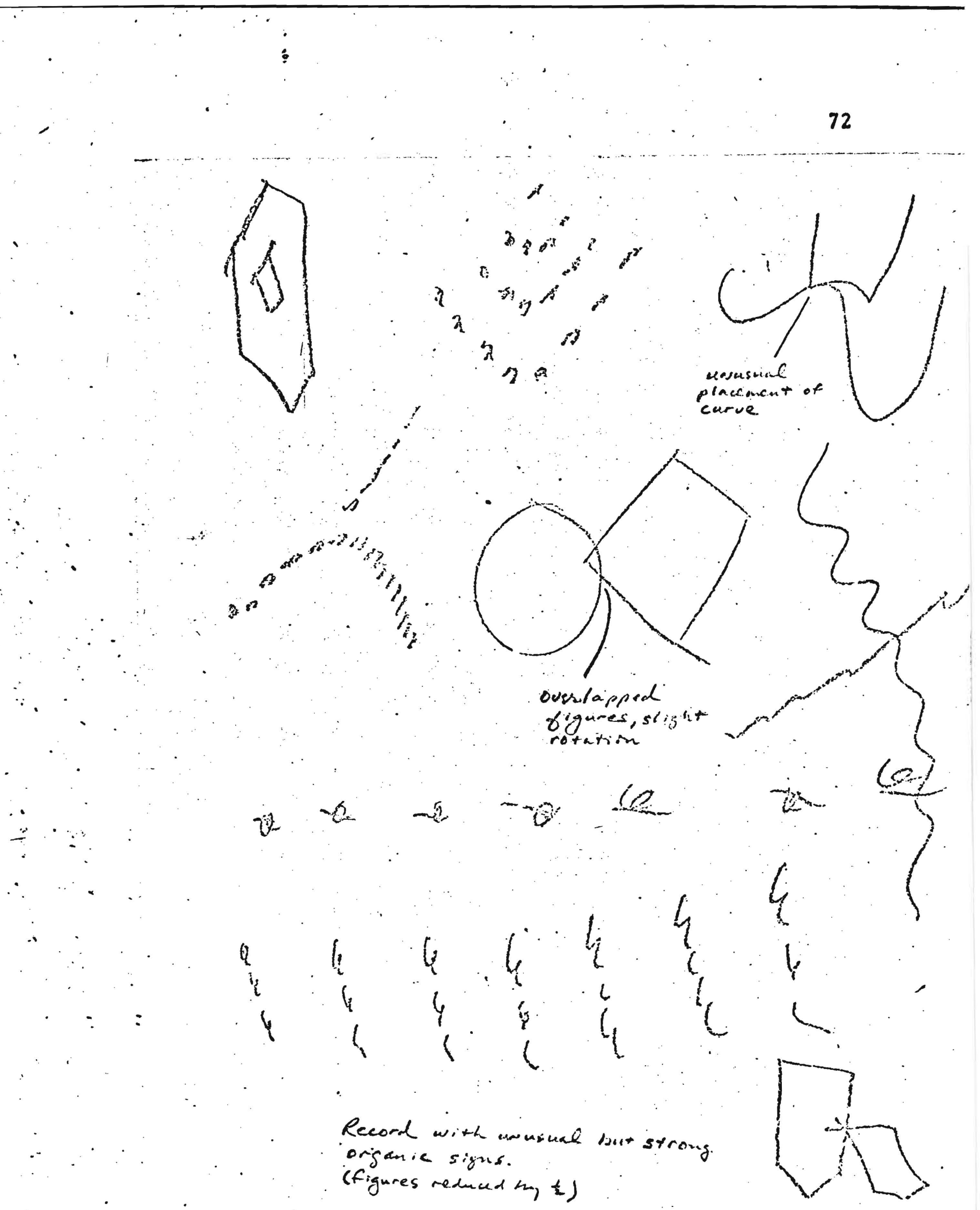


73

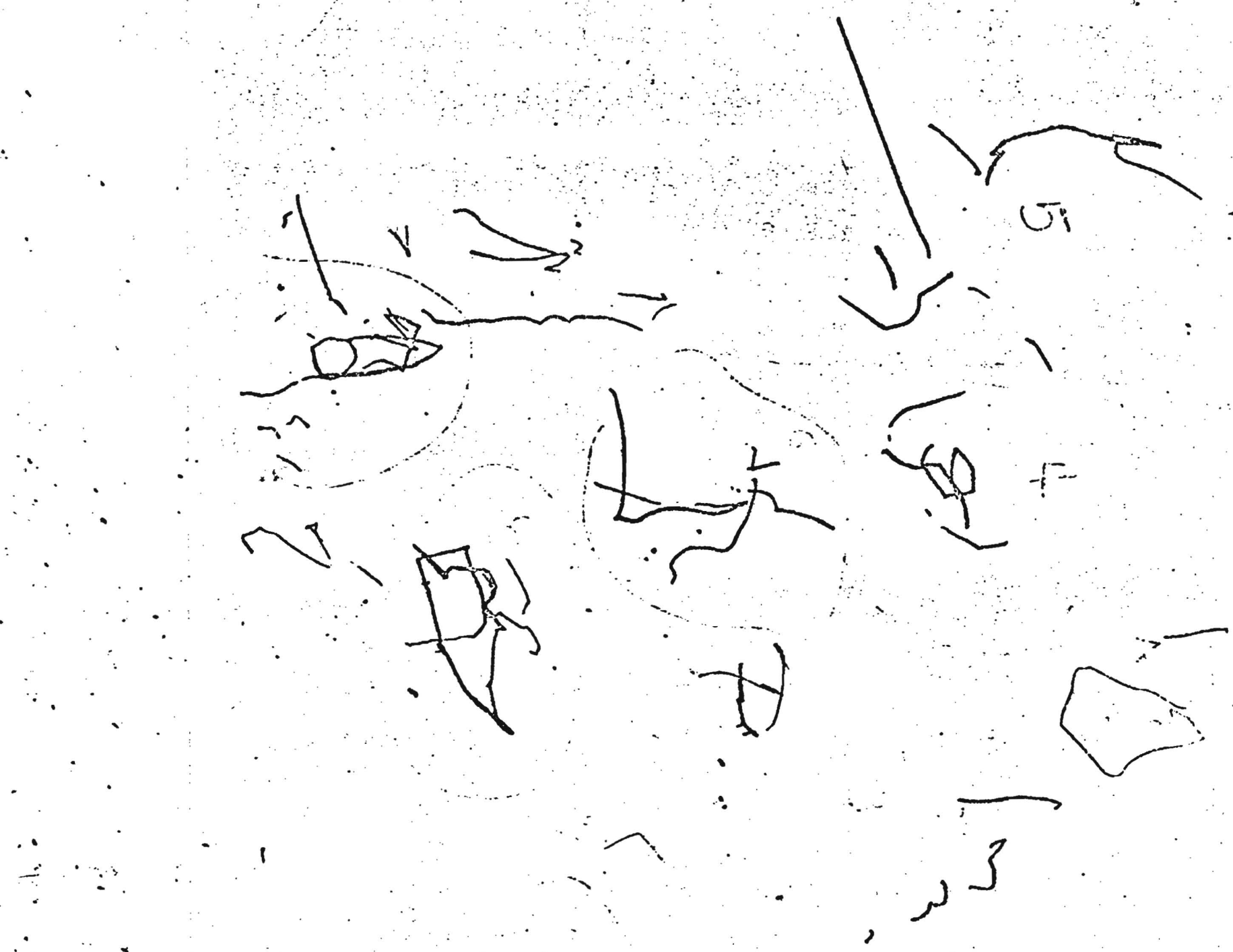

$$
\therefore=-7=1
$$




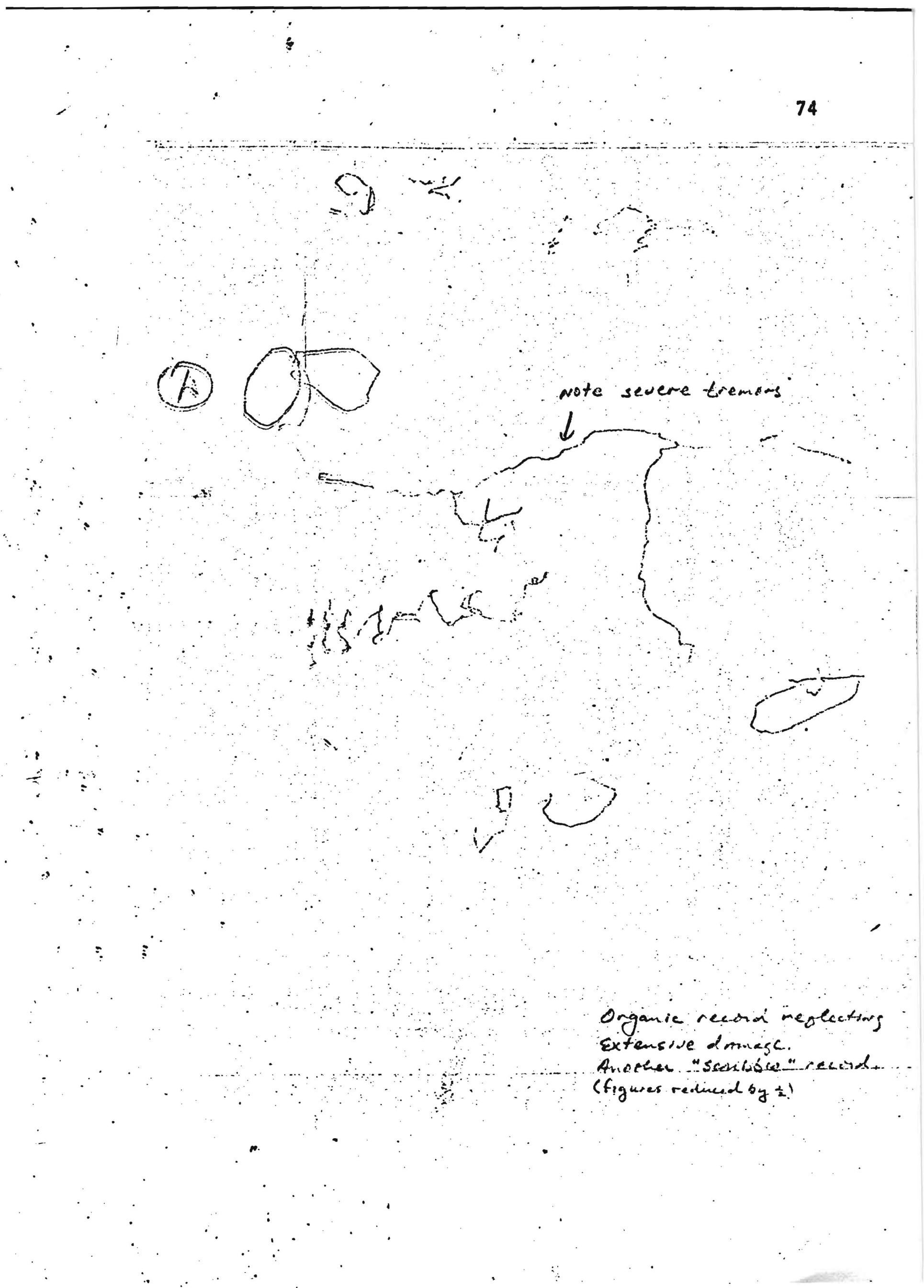


MALINGERERS 
77

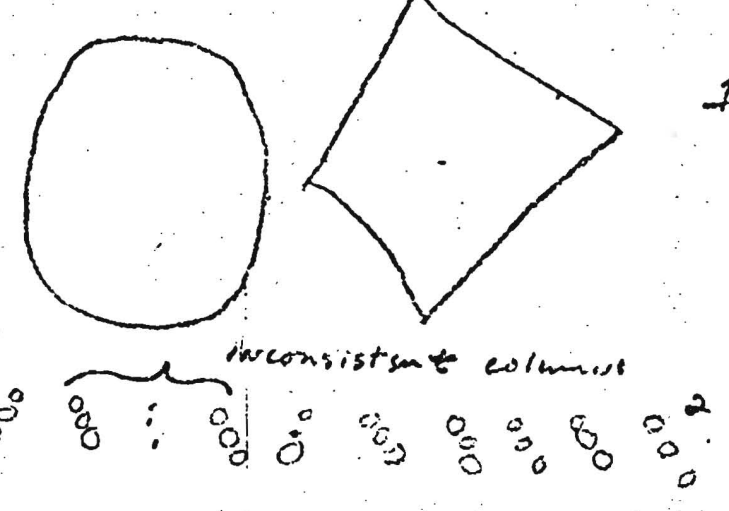

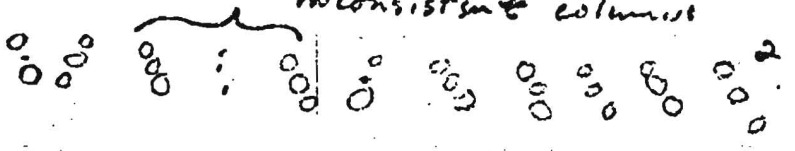

A poor reproduction, especially is comparison to the encoders it Design 2 .
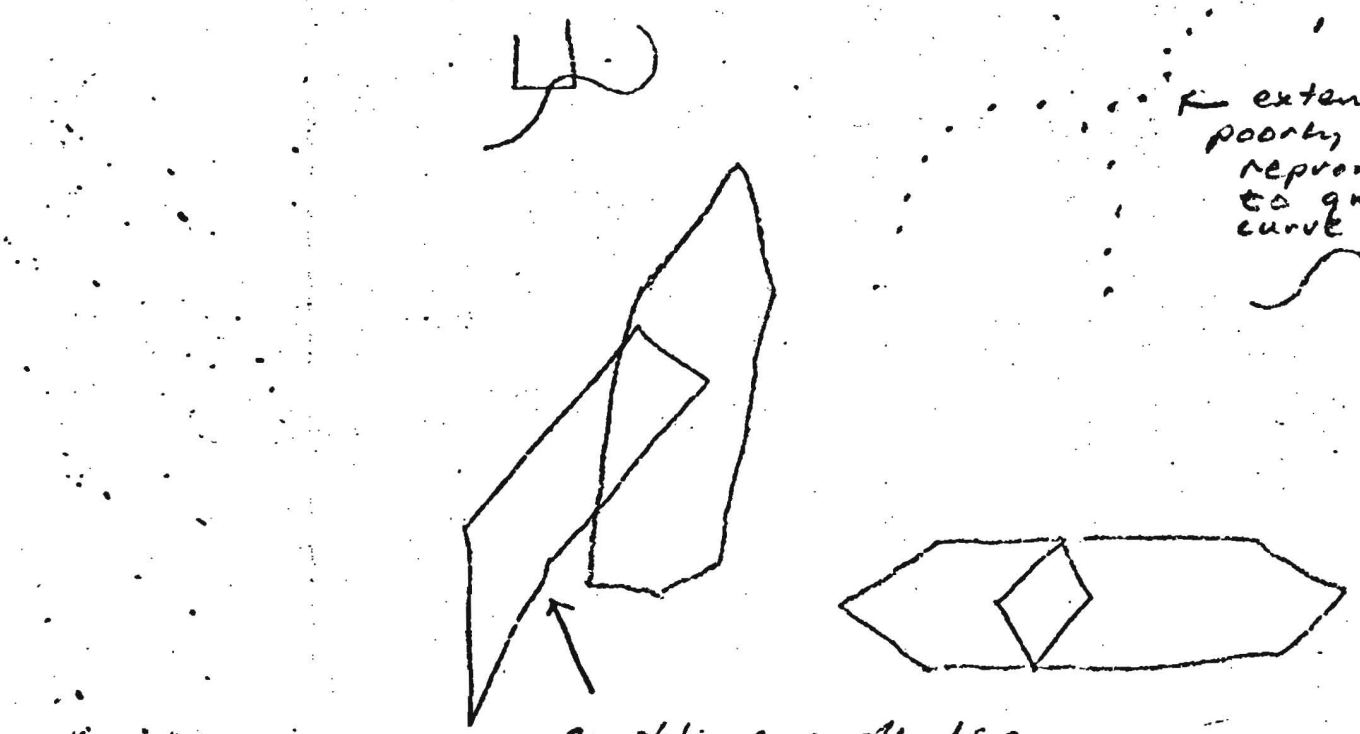

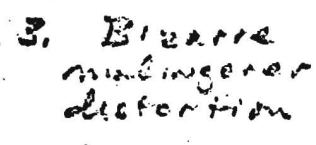

$\because \because \quad$

$\therefore \quad \cdots+\quad \therefore$

an oblique angle lase would be suspect in.

an organic records with

figures of such quality

as 6

(figures reokuced by $\frac{1}{2}$ ) 
$\cdot$

78
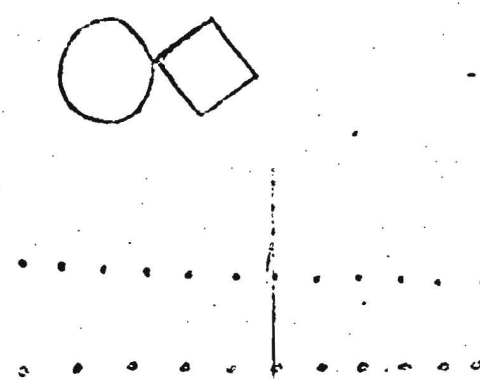

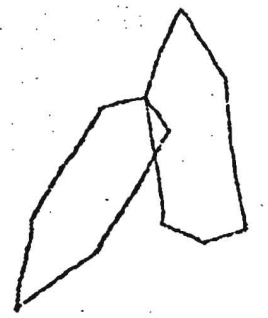

note the eusumess of the tor and beprombuce this forme so accurately

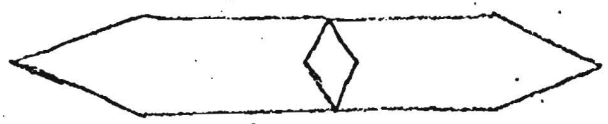

Aside from the tomehpoint, this dicast. is also drain too hackuratery for a. organic 5

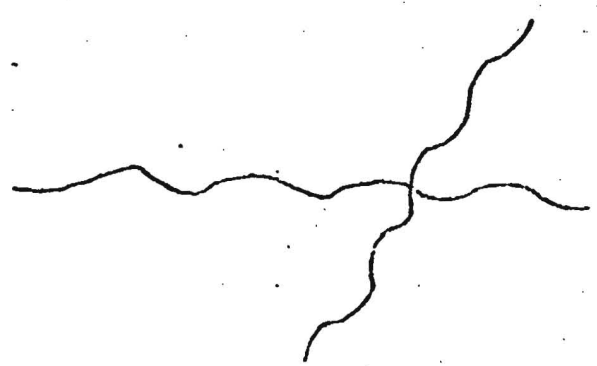

This normal $s$ apposeniry had $1: \pi / 1$ idled about or hat to do when she was instuctal to sucklings r. (figures rededuced by $\frac{1}{2}$ ) 
79 .

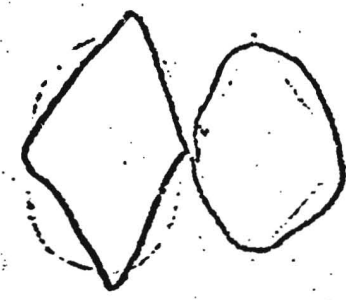

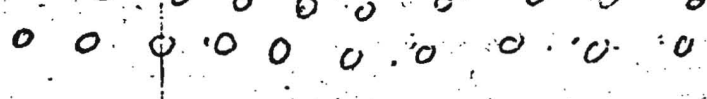

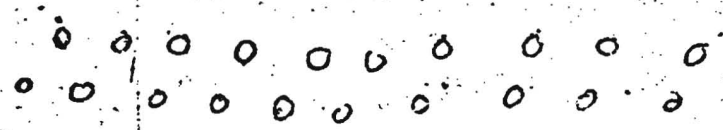

this alosign is tos dueusu relatide to se $0 \quad \therefore 0$

$$
\begin{aligned}
& 00^{0} 0 \\
& 00^{0} \\
& 0
\end{aligned}
$$$$
\therefore 0
$$

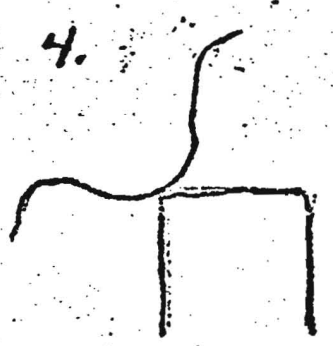

5.

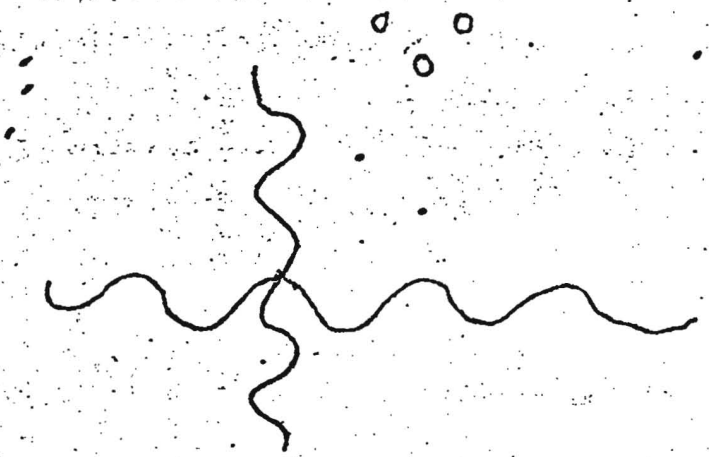

Desigus 4 and 5 che iolatud $100^{\circ}$ - a comancion

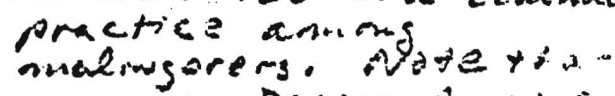
drainas encetb-

B. (figures hove been reduced

B.

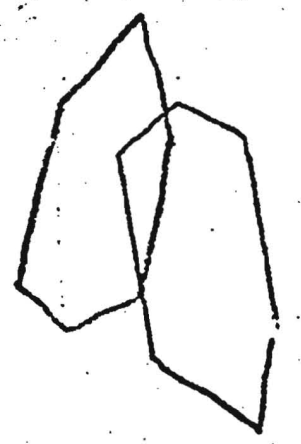
by $\frac{1}{2}$ ) 
$80^{\circ}$

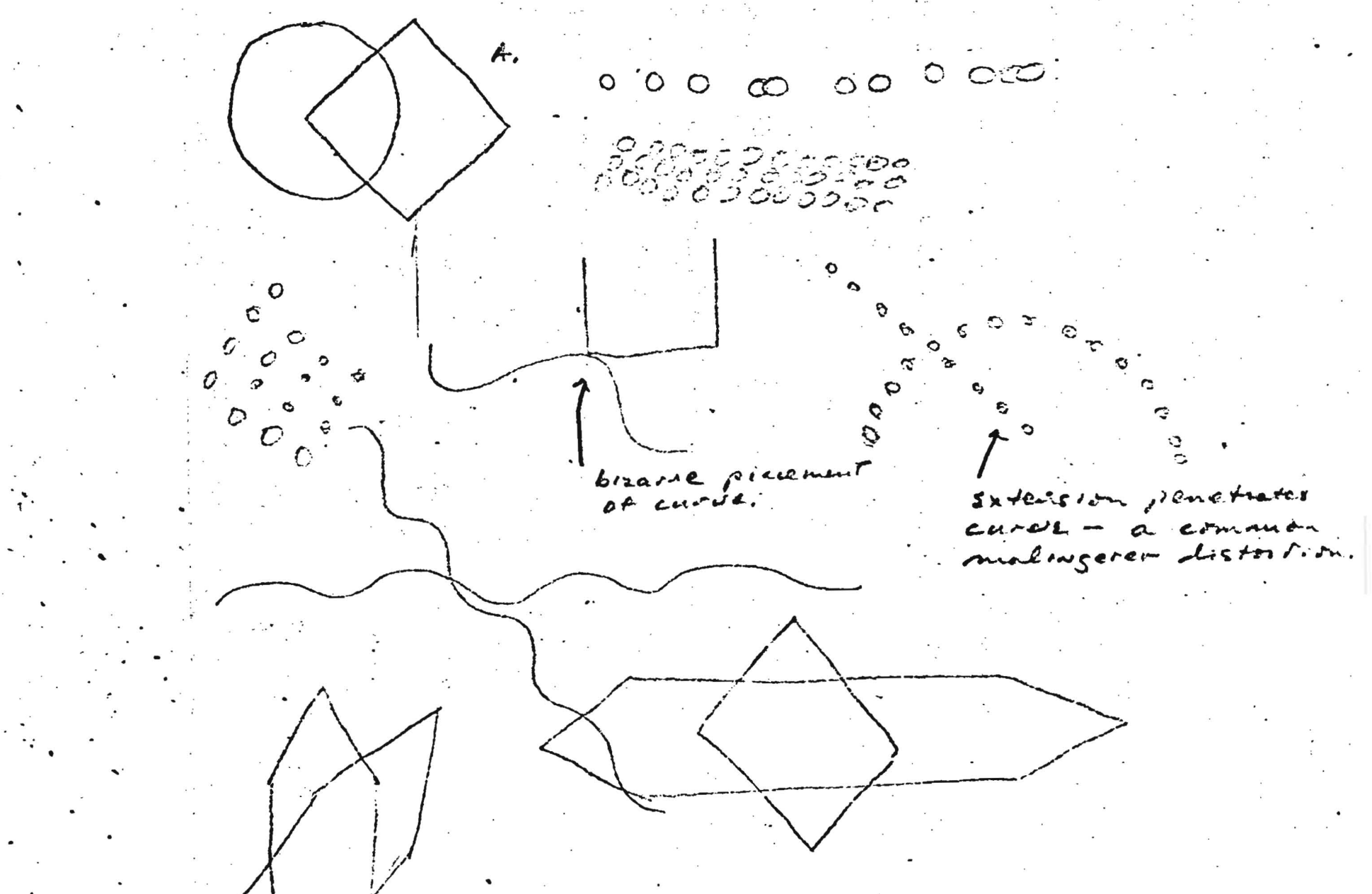

wote hew sxtensively the figurs in Design of overlag. Orgainics sorctetions ouereag these figmes hat ravely to this legree.

(figures reduced in $\frac{1}{3}$ ) 


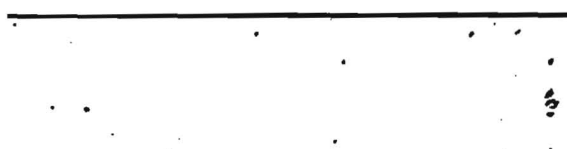

81
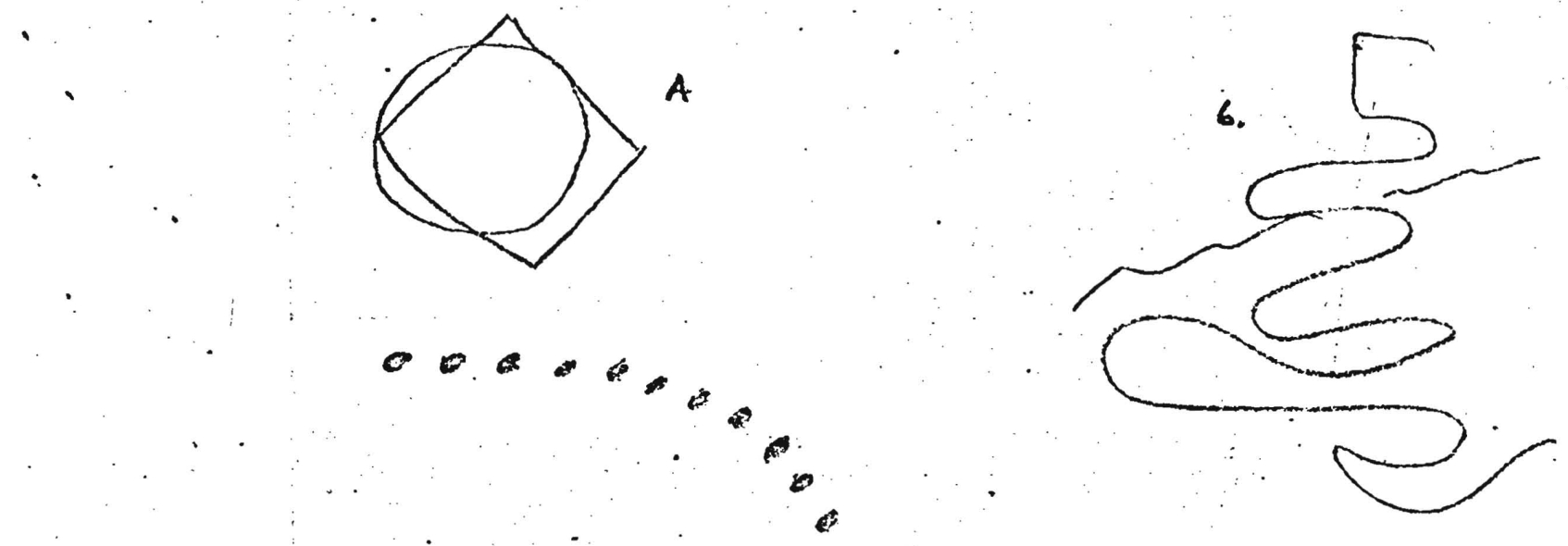
$82^{\circ}$

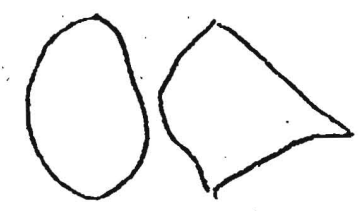

2.

31,1
$1 / 1$
$1 ; 01$
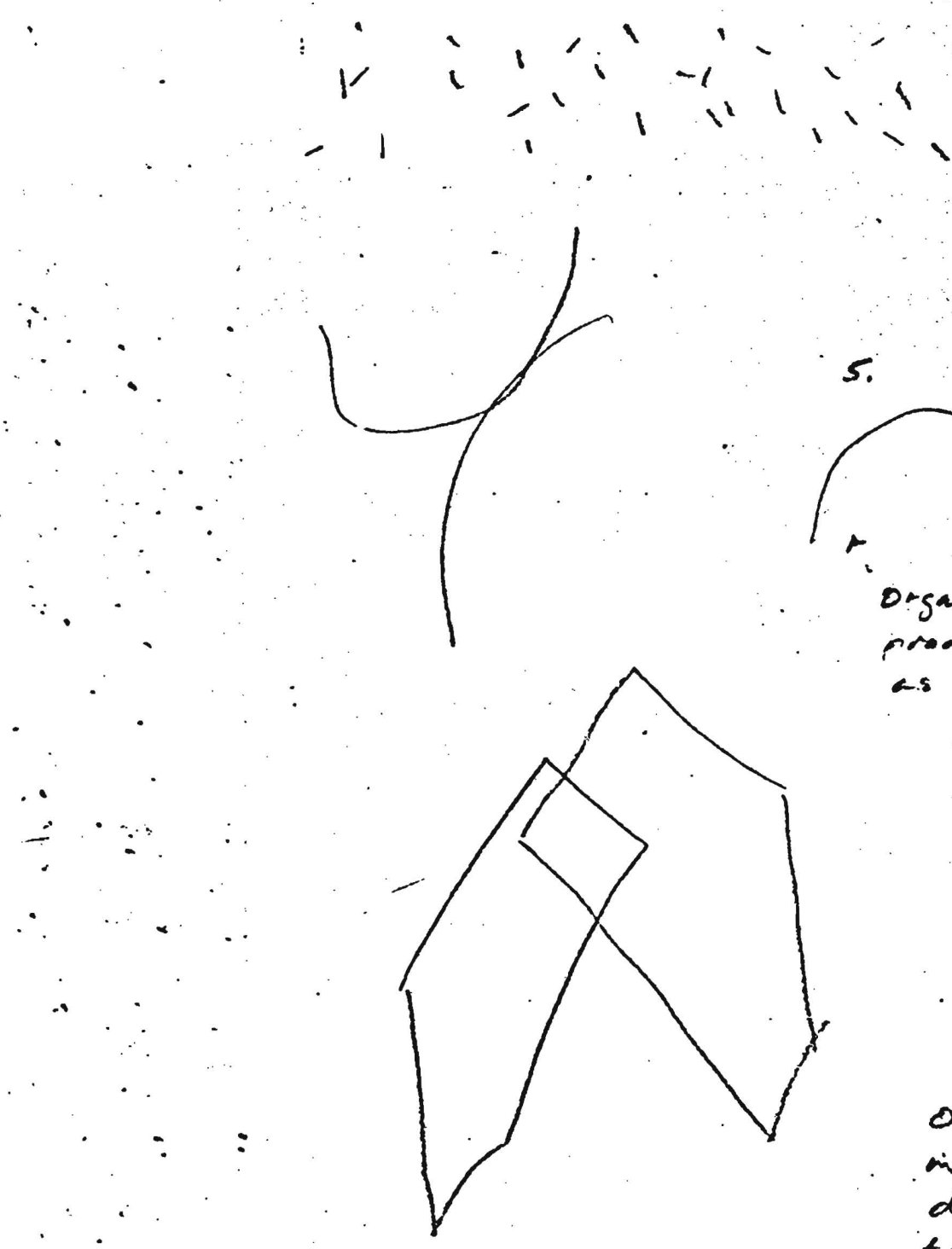

organues aluost mangr as two lines.

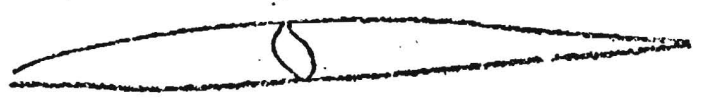

onganics ravel, use slaskes in Desiguis 2 and 5 . If the did, the slaghes wimed tend to be lieson the simme slape.

(figures reduced by $\frac{1}{2}$ ) 

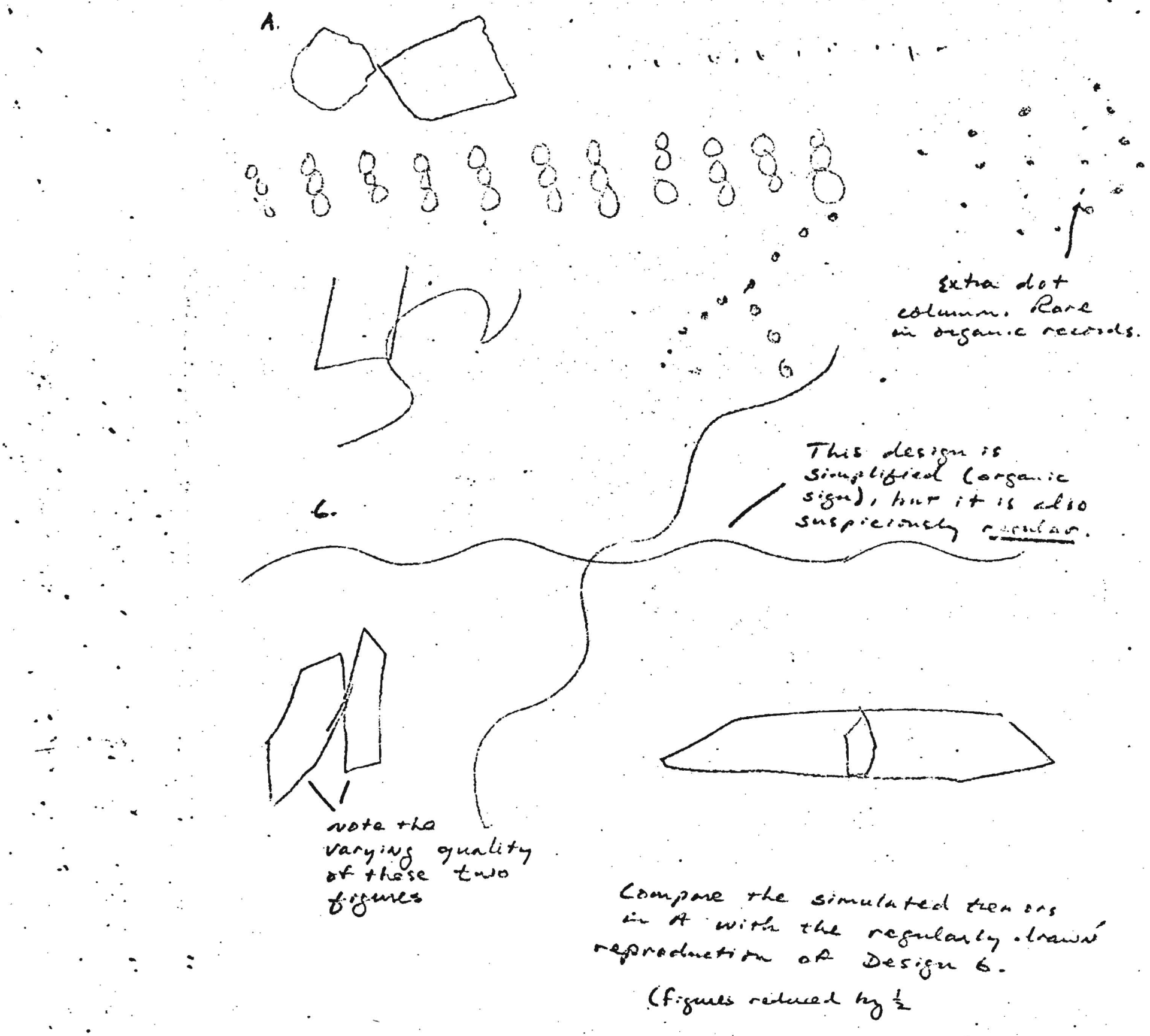
84

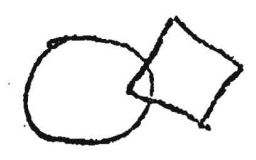

The persavirations on designs land 2 we rane exeept in ongavies with sxtorsinte dormage.

$$
\begin{aligned}
& 060000000000000000000000000000 \\
& 2 \text { o d o } 20000000000000
\end{aligned}
$$

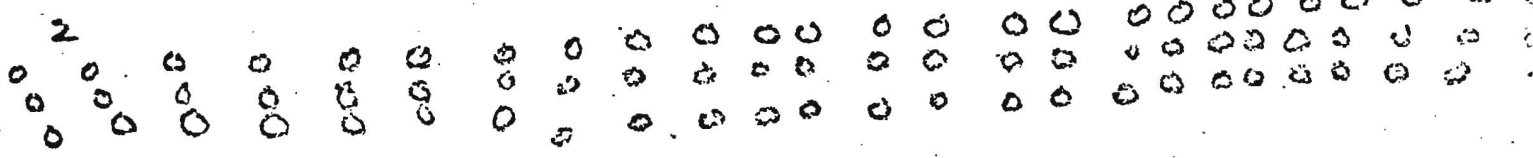
3. $0 \theta_{2} \quad$ The "6"-circles on $0 \cdot 0.00$ suspicasuchy o-ganic. Eut the circlas in Desigus $1+2$ are of muled betien quabity"

$165^{\circ}$ riote haw radeadly the angle shites in these colvemms.

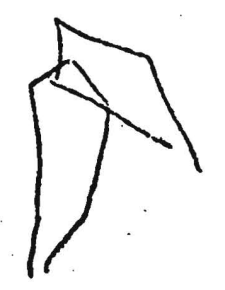

$\because \because \therefore \quad 5000000$

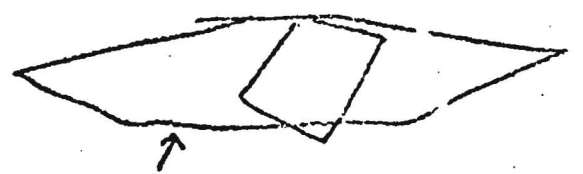

Observe the cintrasucous

flowing ativi in thit tigura - ne signot hesitation disspits

Malingerear recind with orgunin signs. (figures reduced by $\frac{1}{2}$ ) 
85.

t.

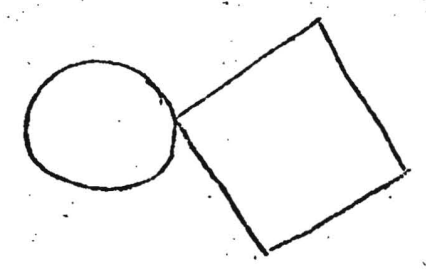

3.

$$
\begin{array}{llllllllll}
0 & 0 & 0 & & & & & & \\
0 & 0 & 0 & 0 & 0 & 0 & 0 & 0 & 0 & 0 \\
0 & 0 & 0 & 0 & 0 & 0 & 0 & 0 & 0 & 0 \\
0 & & 0 & 0 & 0 & 0 & 0 & 0 & 0 & 0
\end{array}
$$

5.<smiles>C#CC#CCC(CCCC)CCCCC</smiles>

9.

8.
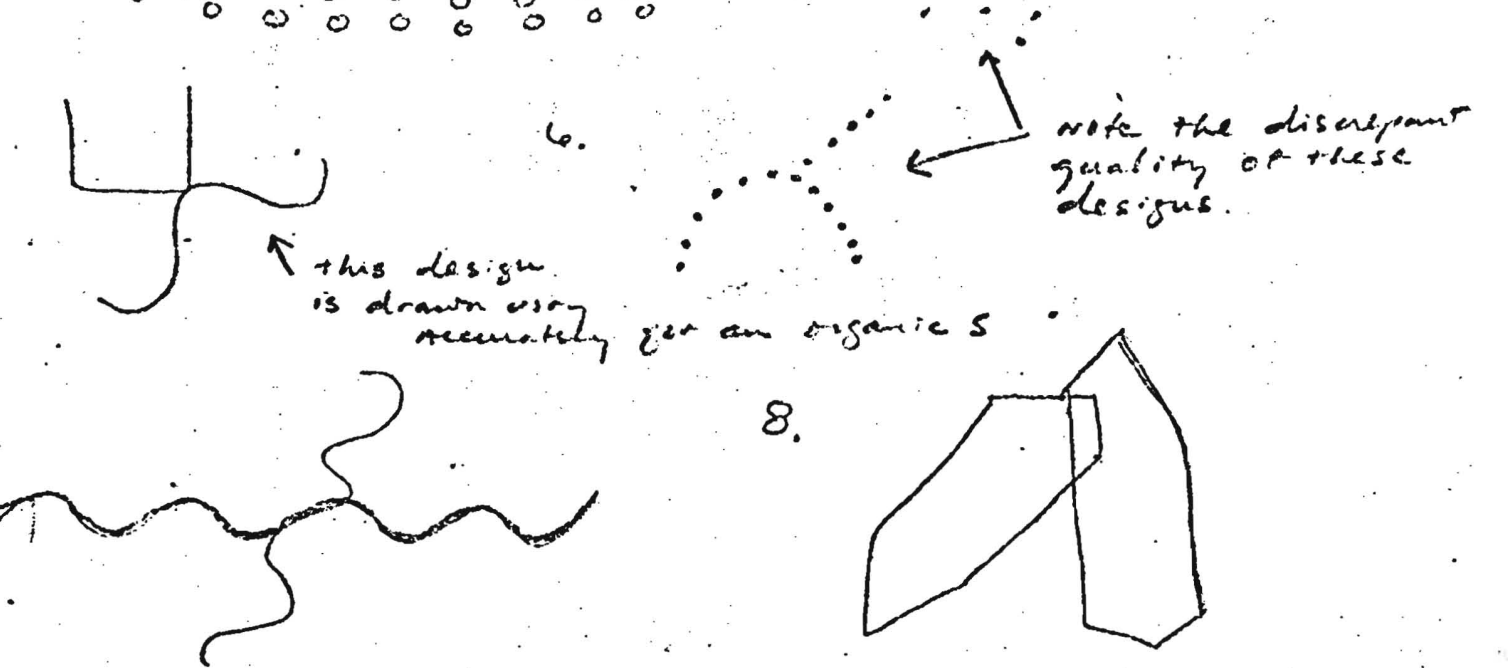

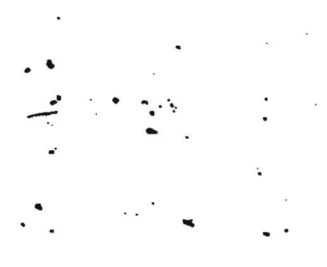

t.

9.<smiles>C1CCC2CCCCC2CC1</smiles>

Notice the ranging

(figures reduced by $\frac{1}{2}$ ) 


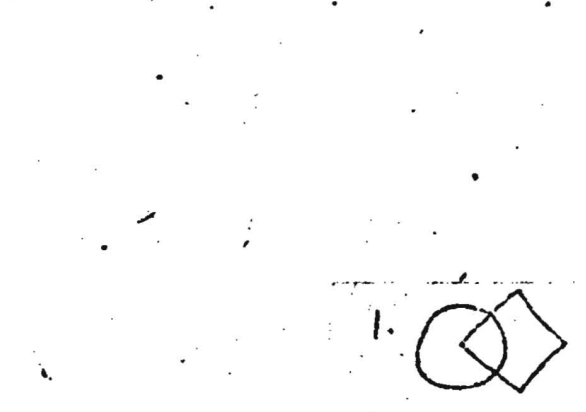

86

1.

2.

3000000000

4. $)\rangle\rangle$ - organics ravecy use linses

5. U

6. $\varnothing$

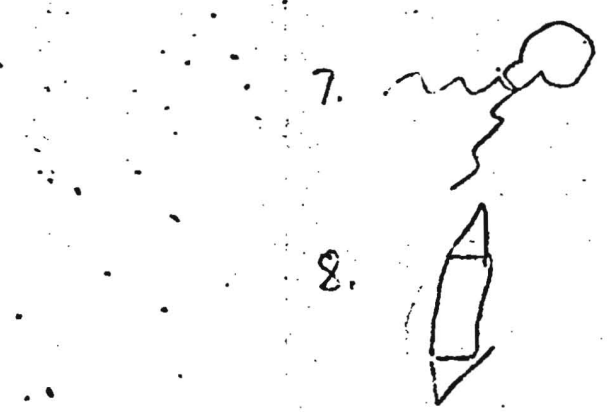

$\because \because \quad \therefore \quad \therefore \quad \therefore \square$

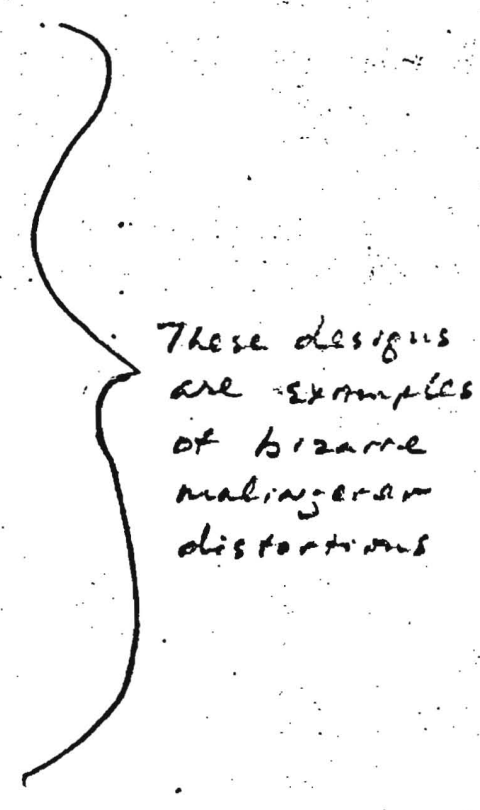

These desigus

of bizarre

malingarsin

(figurest redeced by t) 
$88^{\circ}$
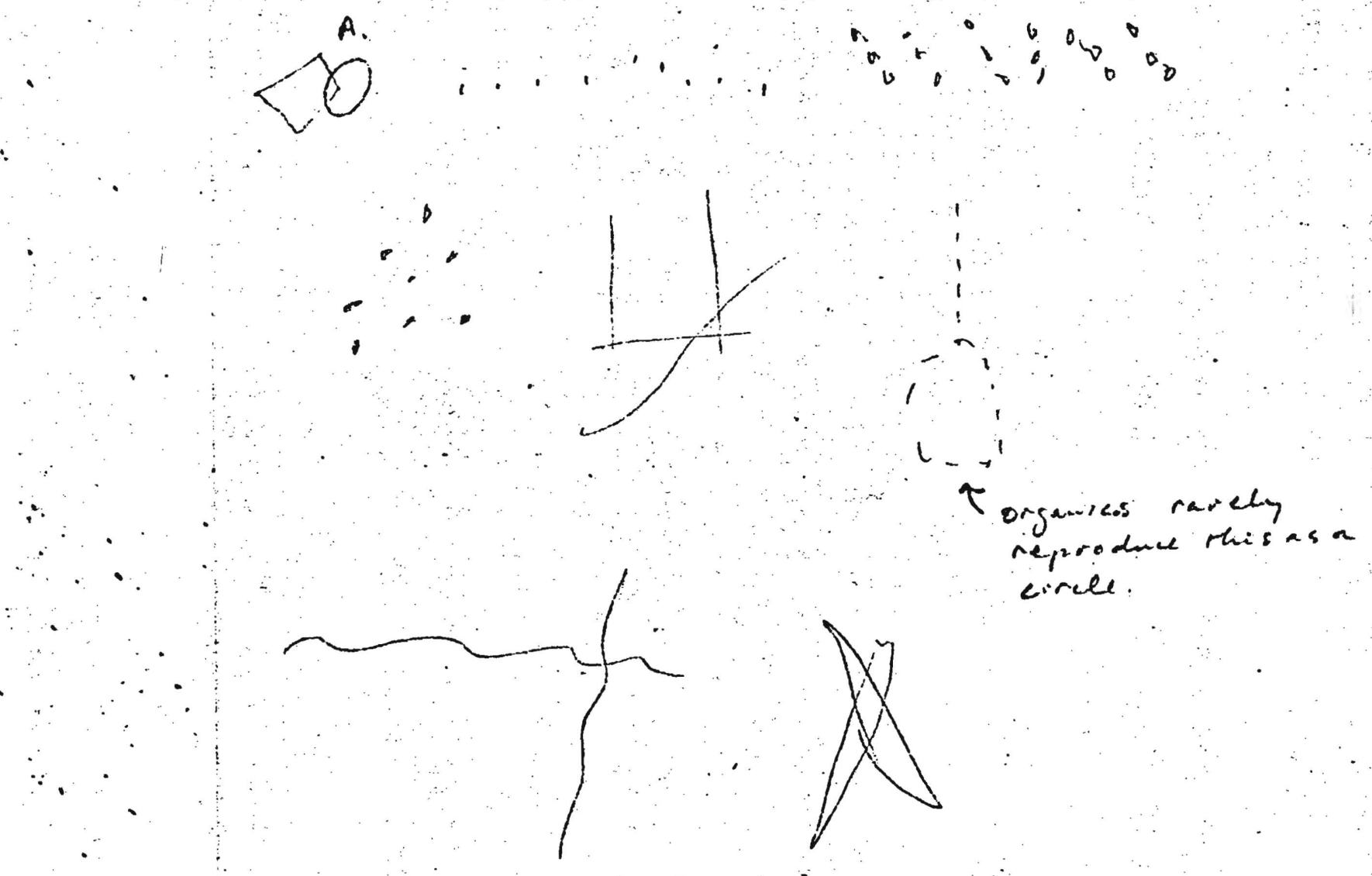
circle.

Design $A$ is the onth ser of figures which is ritarid $180^{\circ}$.

rounded ongles contriturnte to bullit-live shague of

figure. Organices rareeg romud angles in the manoser.

(figures raducad by $\frac{1}{2}$ ) 


\section{APPENDIX F}

THE BENDER-GESTALT TEST FIGURES 